\title{
Isotopologue multi-point calibration for proteomics biomarker quantification in clinical practice
}

\author{
Cristina Chiva ${ }^{1,2}$, Olga Pastor ${ }^{1,2}$, Lucía Trilla-Fuertes ${ }^{3}$, Angelo Gámez-Pozo ${ }^{3,4}$, Juan Ángel Fresno Vara ${ }^{4}$, Eduard
} Sabidó ${ }^{1,2, *}$

${ }^{\text {I} P r o t e o m i c s ~ U n i t, ~ C e n t e r ~ f o r ~ G e n o m i c s ~ R e g u l a t i o n, ~ B a r c e l o n a ~ I n s t i t u t e ~ o f ~ S c i e n c e ~ a n d ~ T e c h n o l o g y ~(B I S T), ~ 08003, ~}$ Barcelona, Spain

${ }^{2}$ Proteomics Unit, Universitat Pompeu Fabra, 08003, Barcelona, Spain

${ }^{3}$ Biomedica Molecular Medicine SL, C/ Faraday 7, 28049, Madrid, Spain

${ }^{4}$ Molecular Oncology \& Pathology Lab, Institute of Medical and Molecular Genetics-INGEMM, La Paz

University Hospital-IdiPAZ, Paseo de la Castellana 261, 28046, Madrid, Spain

* Corresponding author

Eduard Sabidó

Proteomics Unit, Center for Genomics Regulation, Barcelona Institute of Science and Technology (BIST), Universitat Pompeu Fabra, Barcelona, Spain

Dr. Aiguader 88

08003 Barcelona

eduard.sabido@.crg.cat

To editor: Targeted proteomics has become the method of choice for biomarker validation in human biopsies due to its capacity to measure a set of peptides in multiple samples with high sensitivity, reproducibility, accuracy and precision. ${ }^{1-4}$ However, for targeted proteomics technologies to be transferred to clinical routine there is the need to reduce its complexity and make its procedures simpler, increase its throughput and improve its analytical performance. $^{5-7}$

Biomarker peptide quantification with high accuracy and precision is one of the steps that still remains a challenge in clinical routine, mainly due to the difficulty to account for matrix effects (i.e. signal variation due to biological patient variability) and to establish a valid range of quantification for each analyte in each individual sample. ${ }^{8,9}$ Research-grade proteomics laboratories perform targeted peptide quantification with stable isotopically labelled peptides (one per analyte), which are added to the samples and used as internal standards. ${ }^{10,11}$ This strategy, known as single-point calibration, enables to confidently assign the endogenous peptide and infer its quantity by direct comparison to the internal standard. This approach however assumes that both the endogenous and the internal standard peptides are within the linear range of quantification, which is not always granted as the linear dependency between peptide areas and concentrations only occurs in a limited sample-dependent range of concentrations. ${ }^{12}$ Although standard peptide abundances are adjusted to endogenous levels in low-throughput projects, this is not feasible when dealing with large cohorts of patients in clinical routine that exhibit a high variability of peptide abundances and different matrix effects. ${ }^{8,9}$ External multi-point standard curves in their different forms (calibrated, reverse) have been used to alleviate part of these limitations, but the requirement of a representative blank matrix 
and the fact that they do not account for matrix effects in individual samples nor establish a valid range of quantification for each individual sample limit their application. ${ }^{13}$

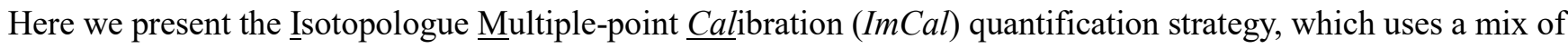
isotopologue peptides to generate internal multiple-point calibration curves for each individual sample and to accurately quantify biomarker peptides in clinical applications without the need of expert supervision. ImCal relies on the use of five different isotopically labelled peptides of different nominal mass-ranging from 10 to $39 \mathrm{Da}$ mass shifts - mixed at different concentrations to be used as internal calibration curve for each endogenous peptide of interest (Figure 1A). Isotopologue peptides have been used in different proteomics applications including the calibration of external dilution curves ${ }^{14}$, the identification of peptide signals and the triggering of subsequent spectra acquisition ${ }^{15}$, the assessment of instrument performance ${ }^{16}$, and the quantification of proteins in highresolution mass spectrometers. ${ }^{17}$ Thus, ImCal expands the use of multiple isotopologue peptides into proteomics quantification and makes it compatible with both high- and low-resolution mass spectrometers. The use of internal multi-point calibration curves is well-suited for the generation of ready-to-use biomarker kits for clinical applications as it is compatible with different levels of endogenous peptide, it eliminates the need for blank matrices required in external curves, it allows the evaluation of matrix effects and the valid quantification range in each individual sample, and does not require expert adjustment. The use of multiple isotopologue peptides in ImCal increases the cost of each targeted assay and limits the number of analytes measured due to cycle time constrains. Although these drawbacks might restrict certain research-grade applications, they are not a significant limitation for clinical routine use in which a very specific set of analytes is systematically quantified in a large number of samples. 
A

\begin{tabular}{|c|c|c|}
\hline $\mathbf{m} / \mathbf{z}$ & Sequence & Amount \\
\hline 483.7482 & ELVSEFSR & unknown \\
\hline 488.7523 & ELVSEFSR[+10] & $200 \mathrm{fmol}$ \\
\hline 491.7592 & ELV [+6]SEFSR[+10] & $100 \mathrm{fmol}$ \\
\hline 495.2678 & $\mathrm{EL}[+7 \mathrm{JV}[+6] \mathrm{SEFSR}[+10]$ & $20 \mathrm{fmol}$ \\
\hline 500.2914 & EL[+7]V[+6]SEF[+10]SR[+10] & $10 \mathrm{fmol}$ \\
\hline 503.2883 & $\mathrm{E}[+6] \mathrm{L}[+7] \mathrm{V}[+6] \mathrm{SEF}[+10] \mathrm{SR}[+10]$ & $2 \mathrm{fmol}$ \\
\hline
\end{tabular}

B

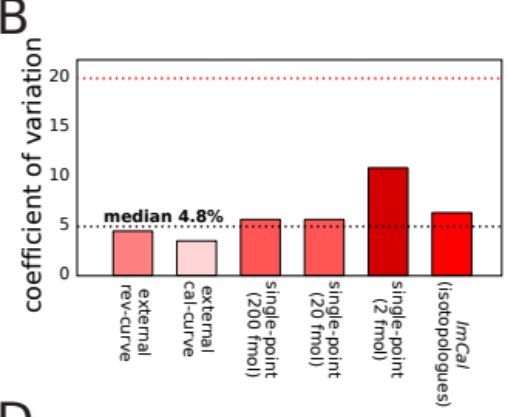

D

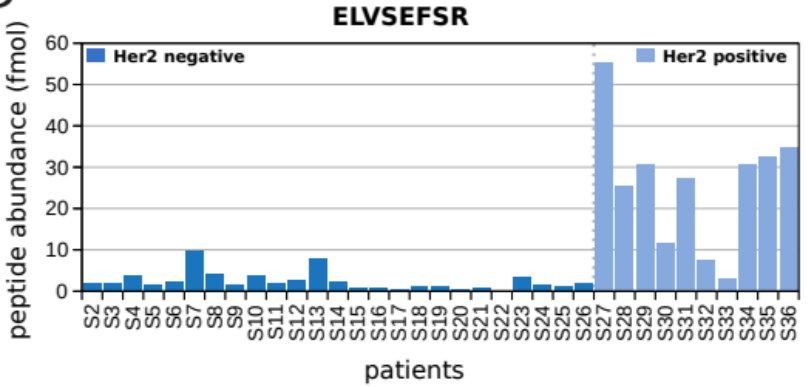

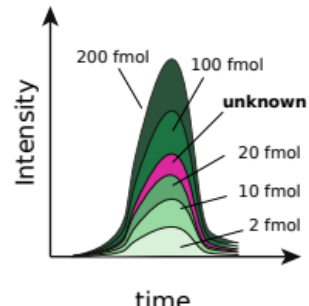

C ELVSEFSR

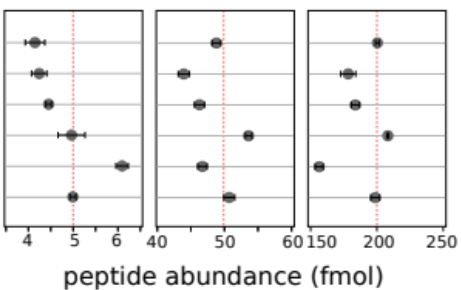

peptide abundance (fmol)

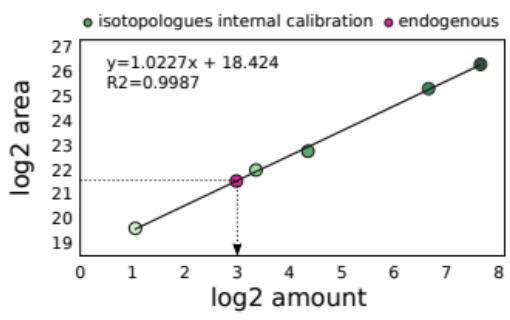

GLQSLPTHDPSPLQR

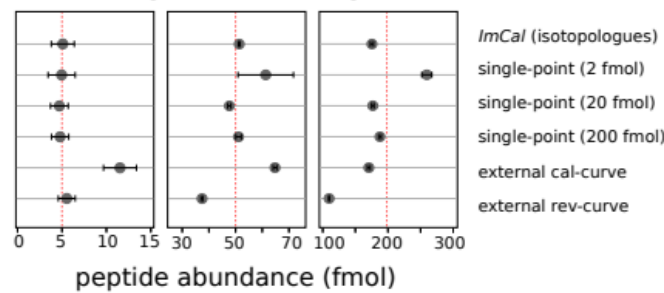

GLQSLPTHDPSPLQR

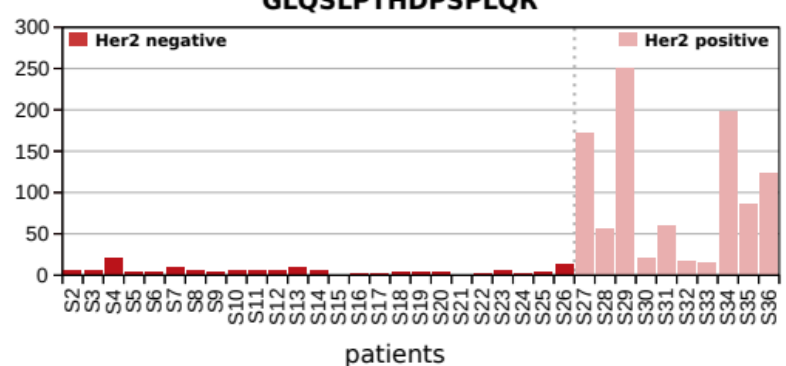

Figure $1 \mathrm{~A})$ Schematic summary of the Isotopologue Multiple-point Calibration (ImCal) quantification strategy with the reference isotopically-labeled peptides represented in green and the targeted endogenous peptide in pink B) Quantification precision in terms of the coefficient of variation obtained in controlled samples with known amounts of targeted peptide (5,50 and $200 \mathrm{fmol})$ using different calibration strategies. C) Quantification accuracy for peptides ELVSEFSR and GLQSLPTHDPSPLQR obtained from controlled samples $(5,50,200$ fmol) with different calibration strategies. D) Calculated abundance of peptides ELVSEFSR and GLQSLPTHDPSPLQR in FFPE samples from Her2+ and Her2- patients.

The performance of ImCal was compared with other quantification strategies, namely single point calibration, external calibrated curves, and reverse calibrated curves by selected reaction monitoring in a triple quadrupole mass spectrometer (Figure 1B-C, Tables S1-S4). For this purpose, two unique peptides for human ERBB2 receptor tyrosine-protein kinase 2 (ELVSEFSR and GLQSLPTHDPSPLQR; Protein HER2, P04626) were selected from the CPTAC assay portal (assays.cancer.gov). ${ }^{18}$ Known amounts (5, 50 and $200 \mathrm{fmol}$ ) of each synthetic nonlabelled peptide were analyzed in a background matrix consisting on a digested $E$. coli protein extract to assess the accuracy and precision of the ImCal method. In parallel, five isotopologues forms were synthesized for each selected peptide using different combinations of ${ }^{13} \mathrm{C}_{6}{ }^{15} \mathrm{~N}_{4}$-Arg, ${ }^{13} \mathrm{C}_{5}{ }^{15} \mathrm{~N}_{1}$-Val, ${ }^{13} \mathrm{C}_{6}{ }^{15} \mathrm{~N}_{1}$-Leu, ${ }^{13} \mathrm{C}_{9}{ }^{15} \mathrm{~N}_{1}-\mathrm{Phe},{ }^{13} \mathrm{C}_{5}{ }^{15} \mathrm{~N}_{1}$ Pro and ${ }^{13} \mathrm{C}_{5}{ }^{15} \mathrm{~N}_{1}$-Glu and mixed at different amounts $(2,10,20,100,200 \mathrm{fmol} / \mu \mathrm{L})$ to generate internal and external calibration curves covering three orders of magnitude (Supplementary Methods). The use of isotopologue multiple-point internal calibration curves resulted in high accuracy and precision for the quantification of the 
selected peptides similar or better than the other peptide quantification strategies evaluated (Figure 1B-C, Figures S1-S2 and Table S4).

Finally, we used the ImCal method to quantify HER2 in formalin-fixed paraffin-embedded (FFPE) tissue biopsies from $10 \mathrm{HER}^{+}$and $25 \mathrm{HER}^{-}$breast cancer patients. HER2 is a membrane-bound receptor from the epidermal growth factor receptor (ERBB) family and it is overexpressed in a significant percentage of breast cancer patients. Therefore, its abundance is used for disease prognosis and patient stratification ${ }^{19,20}$, which makes its quantification via internal multiple-point calibration curves an ideal target for a clinical application of ImCal. Similarly, the use of the $\mathrm{ImCal}$ strategy is particularly relevant in the quantification of peptide biomarkers in FFPE samples. Indeed, FFPE samples are the reference specimens for immunohistochemistry analyses in pathology clinical practice due to its convenience of handling and storage, and its capacity to preserve the physical structure of the tissue. The availability of large archives with rich clinical metadata have also made FFPE samples an attractive source of material for cancer proteomics research ${ }^{21,22}$ and several groups have developed workflows for in-depth proteome analysis of these type of samples. ${ }^{23-25}$ However, FFPE samples present a high variability of peptide signal due to pronounced matrix effects and the linear range of peptide quantification is highly heterogeneous among FFPE samples (Figure S3-S4). Therefore, the use of internal multiple-point calibration curves via the ImCal strategy is of high relevance in peptide biomarker quantification in FFPE samples as it accounts for matrix effects and establishes a valid range of quantification in each individual sample.

Peptide quantification of HER2 in 35 breast cancer patients with the ImCal strategy revealed some signal corresponding to HER2 peptides in patients previously classified as HER2-, as well as a high degree of heterogeneity among HER2+ patients (IHC3+) ranging from values below 5 fmol to values over 250 fmol (Figure 1D, Table S5-S6). In some patients the use of Im Cal curves reveals that endogenous peptide amounts are below the limit of quantification and therefore the reported amounts for these samples might not be as accurate nor precise as the ones within the linear range of quantification. However, the results obtained with the ImCal strategy contrast with the homogeneous immunohistochemistry patient classification and illustrates how an improvement of mass spectrometry methods for biomarker quantification can provide fine-grain patient stratification, and thus better disease diagnostic and prognosis as reported in previous works. ${ }^{26,27}$

Finally, it is noteworthy that despite being fit-for-purpose assays from the CPTAC assay portal, the two selected peptides result in different absolute protein quantification when being used in real patient samples. These results reflect the importance of the protein digestion process, which is affected by the surrounding protein sequence, as well as, by potential post-translational modifications and polymorphisms. For instance, we detect the peptide with a missed cleavage FRELVSEFSR in in-house shotgun analyses of the same patient samples, and despite tryptic digestion being highly reproducible ${ }^{28}$, the presence of different peptidoforms affects in this case the absolute quantification of HER2. Although some of these caveats have been mitigated in the past by the use of isotope labelled protein standards ${ }^{29}$, this observation does not affect the benefits of multi-point internal calibration curves. However, it highlights the importance of peptide selection and its characterization in the protein context when using tryptic peptides as clinical biomarkers.

In conclusion, the use of internal multi-point calibration curves with isotopically labelled peptides of different nominal mass overcomes several of the current limitations for peptide biomarker quantification in clinical proteomics applications. In particular, it is compatible with both high- and low-resolution mass spectrometers as well as with different levels of endogenous peptide. It eliminates the need for blank matrices required in external curves, and it allows the evaluation of matrix effects and definition of a valid quantification range in each individual sample. Therefore, Im Cal is ideal for the development of ready-to-use biomarker kits in samples that 
present variable levels of endogenous peptide due to inherent biological variability or to strong matrix effects such as in FFPE samples. Finally, ImCal simplifies peptide quantification in clinical proteomics and enables for complete integration with existing software analysis tools to automatically identify peptide signals and to establish a valid range of quantification for each sample without expert supervision in clinical routine..$^{30,31}$

The targeted proteomics data have been deposited to the Panorama Public with the dataset identifier PXD012035..$^{32,33}$

\section{Acknowledgments}

The CRG/UPF Proteomics Unit is part of the Spanish Infrastructure for Omics Technologies (ICTS OmicsTech) and it is a member of the ProteoRed PRB3 consortium which is supported by grant PT17/0019 of the PE I $+\mathrm{D}+\mathrm{i}$ 2013-2016 from the Instituto de Salud Carlos III (ISCIII) and ERDF. We acknowledge support from the Spanish Ministry of Science, Innovation and Universities, "Centro de Excelencia Severo Ochoa 2013-2017", SEV-20120208, and "Secretaria d'Universitats i Recerca del Departament d'Economia i Coneixement de la Generalitat de Catalunya" (2017SGR595). This project has also received funding from the European Union's Horizon 2020 research and innovation program under grant agreement No 823839 (EPIC-XS). LT-F is supported by Spanish Economy and Competitiveness Ministry (DI-15-07614). Molecular Oncology and Pathology Lab is supported by Instituto de Salud Carlos III, Spanish Economy and Competitiveness Ministry, Spain and co-funded by the FEDER program, "Una forma de hacer Europa" (PI15/01310).

\section{Keywords}

Clinical proteomics, biomarkers, isotopologue, internal calibration, protein quantification.

\section{Supporting Information Available}

Supplementary Figures S1-S4

Supplementary Tables S1-S6

Supplementary Methods

- Isotopologue peptides

- Background matrix preparation

- Calibration curves preparation

- Control sample preparation

- Formalin-fixed paraffin-embedded sample preparation

- Data acquisition

- Bibliography 


\section{Bibliography}

(1) Kennedy, J. J.; Abbatiello, S. E.; Kim, K.; Yan, P.; Whiteaker, J. R.; Lin, C.; Kim, J. S.; Zhang, Y.; Wang, X.; Ivey, R. G.; et al. Demonstrating the Feasibility of Large-Scale Development of Standardized Assays to Quantify Human Proteins. Nat. Methods 2014, 11 (2), 149-155. https://doi.org/10.1038/nmeth.2763. Abbatiello, S. E.; Schilling, B.; Mani, D. R.; Zimmerman, L. J.; Hall, S. C.; MacLean, B.; Albertolle, M.; Allen, S.; Burgess, M.; Cusack, M. P.; et al. Large-Scale Interlaboratory Study to Develop, Analytically Validate and Apply Highly Multiplexed, Quantitative Peptide Assays to Measure Cancer-Relevant Proteins in Plasma. Mol. Cell. Proteomics MCP 2015, 14 (9), 2357-2374. https://doi.org/10.1074/mcp.M114.047050.

(3) Borràs, E.; Cantó, E.; Choi, M.; Villar, L. M.; Álvarez-Cermeño, J. C.; Chiva, C.; Montalban, X.; Vitek, O.; Comabella, M.; Sabidó, E. Protein-Based Classifier to Predict Conversion from Clinically Isolated Syndrome to Multiple Sclerosis. Mol. Cell. Proteomics 2016, 15 (1), 318-328.

(4) Sequeiros, T.; Rigau, M.; Chiva, C.; Montes, M.; Garcia-Grau, I.; Garcia, M.; Diaz, S.; Celma, A.; Bijnsdorp, I.; Campos, A. Targeted Proteomics in Urinary Extracellular Vesicles Identifies Biomarkers for Diagnosis and Prognosis of Prostate Cancer. Oncotarget 2017, 8 (3), 4960.

(5) Bache, N.; Geyer, P. E.; Bekker-Jensen, D. B.; Hoerning, O.; Falkenby, L.; Treit, P. V.; Doll, S.; Paron, I.; Müller, J. B.; Meier, F.; et al. A Novel LC System Embeds Analytes in Pre-Formed Gradients for Rapid, Ultra-Robust Proteomics. Mol. Cell. Proteomics MCP 2018. https://doi.org/10.1074/mcp.TIR118.000853.

(6) Abbatiello, S. E.; Mani, D. R.; Schilling, B.; Maclean, B.; Zimmerman, L. J.; Feng, X.; Cusack, M. P.; Sedransk, N.; Hall, S. C.; Addona, T.; et al. Design, Implementation and Multisite Evaluation of a System Suitability Protocol for the Quantitative Assessment of Instrument Performance in Liquid ChromatographyMultiple Reaction Monitoring-MS (LC-MRM-MS). Mol. Cell. Proteomics MCP 2013, 12 (9), 2623-2639. https://doi.org/10.1074/mcp.M112.027078.

(7) Carr, S. A.; Abbatiello, S. E.; Ackermann, B. L.; Borchers, C.; Domon, B.; Deutsch, E. W.; Grant, R. P.; Hoofnagle, A. N.; Hüttenhain, R.; Koomen, J. M.; et al. Targeted Peptide Measurements in Biology and Medicine: Best Practices for Mass Spectrometry-Based Assay Development Using a Fit-for-Purpose Approach. Mol. Cell. Proteomics MCP 2014, 13 (3), 907-917. https://doi.org/10.1074/mcp.M113.036095. Tang, L.; Kebarle, P. Dependence of Ion Intensity in Electrospray Mass Spectrometry on the Concentration of the Analytes in the Electrosprayed Solution. Anal. Chem. 1993, 65 (24), 3654-3668. https://doi.org/10.1021/ac00072a020.

(9) Taylor, P. J. Matrix Effects: The Achilles Heel of Quantitative High-Performance Liquid ChromatographyElectrospray-Tandem Mass Spectrometry. Clin. Biochem. 2005, 38 (4), 328-334. https://doi.org/10.1016/j.clinbiochem.2004.11.007.

(10) Gerber, S. A.; Rush, J.; Stemman, O.; Kirschner, M. W.; Gygi, S. P. Absolute Quantification of Proteins and Phosphoproteins from Cell Lysates by Tandem MS. Proc. Natl. Acad. Sci. 2003, 100 (12), 6940-6945. https://doi.org/10.1073/pnas.0832254100.

(11) Anderson, L.; Hunter, C. L. Quantitative Mass Spectrometric Multiple Reaction Monitoring Assays for Major Plasma Proteins. Mol. Cell. Proteomics 2006, 5 (4), 573-588. https://doi.org/10.1074/mcp.M500331-MCP200.

(12) Bondarenko, P. V.; Chelius, D.; Shaler, T. A. Identification and Relative Quantitation of Protein Mixtures by Enzymatic Digestion Followed by Capillary Reversed-Phase Liquid Chromatography-Tandem Mass Spectrometry. Anal. Chem. 2002, 74 (18), 4741-4749.

(13) Campbell, J.; Rezai, T.; Prakash, A.; Krastins, B.; Dayon, L.; Ward, M.; Robinson, S.; Lopez, M. Evaluation of Absolute Peptide Quantitation Strategies Using Selected Reaction Monitoring. PROTEOMICS 2011, 11 (6), 1148-1152. https://doi.org/10.1002/pmic.201000511.

(14) LeBlanc, A.; Michaud, S. A.; Percy, A. J.; Hardie, D. B.; Yang, J.; Sinclair, N. J.; Proudfoot, J. I.; Pistawka, A.; Smith, D. S.; Borchers, C. H. Multiplexed MRM-Based Protein Quantitation Using Two Different Stable Isotope-Labeled Peptide Isotopologues for Calibration. J. Proteome Res. 2017, 16 (7), 2527-2536.

Gallien, S.; Kim, S.-Y.; Domon, B. Large-Scale Targeted Proteomics Using Internal Standard TriggeredParallel Reaction Monitoring. Mol. Cell. Proteomics $M C P \quad 2015$. https://doi.org/10.1074/mcp.O114.043968. 
(16) Beri, J.; Rosenblatt, M. M.; Strauss, E.; Urh, M.; Bereman, M. S. Reagent for Evaluating Liquid Chromatography-Tandem Mass Spectrometry (LC-MS/MS) Performance in Bottom-Up Proteomic Experiments. Anal. Chem. 2015, 87 (23), 11635-11640. https://doi.org/10.1021/acs.analchem.5b04121.

(17) Hermanson, G.; Rogers, J. C.; Louette, J.; Bomgarden, R. D.; Patel, B. Absolute Quantitation of Proteins and Protein Modifications by Mass Spectrometry with Multiplexed Internal Standards. US9252003B2, February 2, 2016.

(18) Whiteaker, J. R.; Halusa, G. N.; Hoofnagle, A. N.; Sharma, V.; MacLean, B.; Yan, P.; Wrobel, J. A.; Kennedy, J.; Mani, D. R.; Zimmerman, L. J.; et al. CPTAC Assay Portal: A Repository of Targeted Proteomic Assays. Nat. Methods 2014, 11 (7), 703-704. https://doi.org/10.1038/nmeth.3002.

(19) Giordano, S. H.; Temin, S.; Kirshner, J. J.; Chandarlapaty, S.; Crews, J. R.; Davidson, N. E.; Esteva, F. J.; Gonzalez-Angulo, A. M.; Krop, I.; Levinson, J.; et al. Systemic Therapy for Patients With Advanced Human Epidermal Growth Factor Receptor 2-Positive Breast Cancer: American Society of Clinical Oncology Clinical Practice Guideline. J. Clin. Oncol. 2014, 32 (19), 2078-2099. https://doi.org/10.1200/JCO.2013.54.0948.

(20) Wolff, A. C.; Hammond, M. E.; Schwartz, J. N.; Hagerty, K. L.; Allred, D. C.; Cote, R. J.; Dowsett, M.; Fitzgibbons, P. L.; Hanna, W. M.; Langer, A. College of American Pathologists American Society of Clinical Oncology/College of American Pathologists Guideline Recommendations for Human Epidermal Growth Factor Receptor 2 Testing in Breast Cancer. J Clin Oncol 2007, 25 (1), 118-145.

(21) Gámez-Pozo, A.; Trilla-Fuertes, L.; Prado-Vázquez, G.; Chiva, C.; López-Vacas, R.; Nanni, P.; BergesSoria, J.; Grossmann, J.; Díaz-Almirón, M.; Ciruelos, E.; et al. Prediction of Adjuvant Chemotherapy Response in Triple Negative Breast Cancer with Discovery and Targeted Proteomics. PloS One 2017, 12 (6), e0178296. https://doi.org/10.1371/journal.pone.0178296.

(22) Wiśniewski, J. R.; Ostasiewicz, P.; Duś, K.; Zielińska, D. F.; Gnad, F.; Mann, M. Extensive Quantitative Remodeling of the Proteome between Normal Colon Tissue and Adenocarcinoma. Mol. Syst. Biol. 2012, 8, 611. https://doi.org/10.1038/msb.2012.44.

(23) Wiśniewski, J. R.; Ostasiewicz, P.; Mann, M. High Recovery FASP Applied to the Proteomic Analysis of Microdissected Formalin Fixed Paraffin Embedded Cancer Tissues Retrieves Known Colon Cancer Markers. J. Proteome Res. 2011, 10 (7), 3040-3049. https://doi.org/10.1021/pr200019m.

(24) Wiśniewski, J. R.; Duś, K.; Mann, M. Proteomic Workflow for Analysis of Archival Formalin-Fixed and Paraffin-Embedded Clinical Samples to a Depth of 10000 Proteins. PROTEOMICS-Clinical Appl. 2013, 7 (3-4), 225-233.

(25) Ostasiewicz, P.; Zielinska, D. F.; Mann, M.; Wiśniewski, J. R. Proteome, Phosphoproteome, and NGlycoproteome Are Quantitatively Preserved in Formalin-Fixed Paraffin-Embedded Tissue and Analyzable by High-Resolution Mass Spectrometry. J. Proteome Res. 2010, 9 (7), 3688-3700.

(26) Nuciforo, P.; Thyparambil, S.; Aura, C.; Garrido-Castro, A.; Vilaro, M.; Peg, V.; Jimenez, J.; Vicario, R.; Cecchi, F.; Hoos, W.; et al. High HER2 Protein Levels Correlate with Increased Survival in Breast Cancer Patients Treated with Anti-HER2 Therapy. Mol. Oncol. 2016, 10 (1), 138-147. https://doi.org/10.1016/j.molonc.2015.09.002.

Nuciforo, P.; Aura, C.; Thyparambil, S. P.; Vilaro, M.; Garrido-Castro, A. C.; Peg, V.; Jimenez, J.; Hoos, W. A.; Burrows, J.; Hembrough, T. A.; et al. HER2 Quantification by Mass Spectrometry Compared to IHC or ISH in Predicting Clinical Benefit from Anti-HER2 Therapy in HER2-Positive Breast Cancer (BC). J. Clin. Oncol. 2015, 33 (15 suppl), 605-605. https://doi.org/10.1200/jco.2015.33.15 suppl.605.

(28) Chiva, C.; Sabidó, E. Peptide Selection for Targeted Protein Quantitation. J. Proteome Res. 2017, 16 (3), 1376-1380. https://doi.org/10.1021/acs.jproteome.6b00115.

(29) Brun, V.; Dupuis, A.; Adrait, A.; Marcellin, M.; Thomas, D.; Vandenesch, F.; Garin, J. Isotope-Labeled Protein Standards toward Absolute Quantitative Proteomics. Mol. Cell. Proteomics 2007, 6 (12), 2139 2149.

(30) Galitzine, C.; Egertson, J.; Abbatiello, S. E.; Henderson, C. M.; Pino, L.; MacCoss, M. J.; Hoofnagle, A. N.; Vitek, O. Nonlinear Regression Improves Accuracy of Characterization of Multiplexed Mass Spectrometric Assays. Mol. Cell. Proteomics 2018, mcp-RA117.

(31) Reiter, L.; Rinner, O.; Picotti, P.; Hüttenhain, R.; Beck, M.; Brusniak, M.-Y.; Hengartner, M. O.; Aebersold, R. MProphet: Automated Data Processing and Statistical Validation for Large-Scale SRM Experiments. Nat. Methods 2011, 8 (5), 430. 
(32) Sharma, V.; Eckels, J.; Schilling, B.; Ludwig, C.; Jaffe, J. D.; MacCoss, M. J.; MacLean, B. Panorama Public: A Public Repository for Quantitative Data Sets Processed in Skyline. Mol. Cell. Proteomics 2018, $17(6), 1239-1244$.

(33) Vizcaíno, J. A.; Deutsch, E. W.; Wang, R.; Csordas, A.; Reisinger, F.; Ríos, D.; Dianes, J. A.; Sun, Z.; Farrah, T.; Bandeira, N.; et al. ProteomeXchange Provides Globally Coordinated Proteomics Data Submission and Dissemination. Nat. Biotechnol. 2014, 32 (3), 223-226. https://doi.org/10.1038/nbt.2839. 


\section{5 fmol - GLQSLPTHDPSPLQR}

Replicate 1

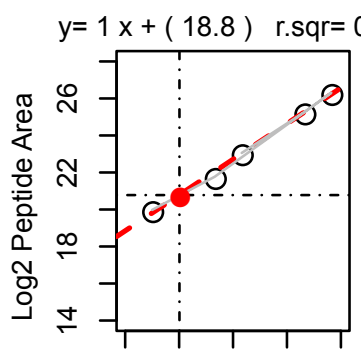

$\begin{array}{lllll}0 & 2 & 4 & 6 & 8\end{array}$

Log2 Quantity (fmol)
Replicate 2

$y=1 x+(18.9) \quad r . s q r=1$

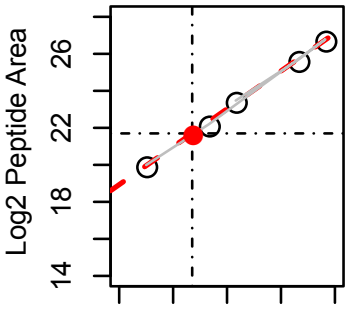

$\begin{array}{lllll}0 & 2 & 4 & 6 & 8\end{array}$

Log2 Quantity (fmol)
Replicate 3

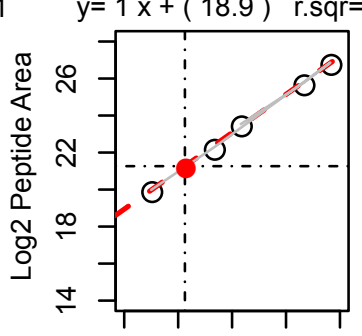

$\begin{array}{lllll}0 & 2 & 4 & 6 & 8\end{array}$ Log2 Quantity (fmol)

\section{0 fmol - GLQSLPTHDPSPLQR}

Replicate 1

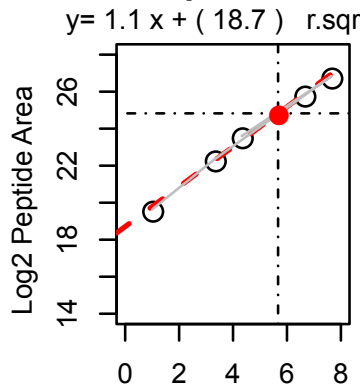

Log2 Quantity (fmol)
Replicate 2

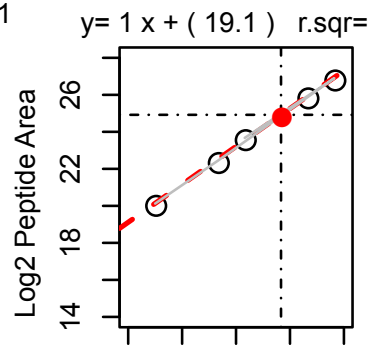

$\begin{array}{lllll}0 & 2 & 4 & 6 & 8\end{array}$ Log2 Quantity (fmol)
Replicate $\mathbf{3}$

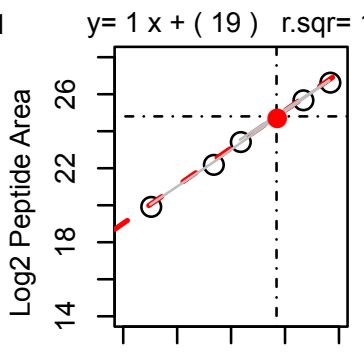

$\begin{array}{lllll}0 & 2 & 4 & 6 & 8\end{array}$ Log2 Quantity (fmol)

\section{$200 \mathrm{fmol}$ - GLQSLPTHDPSPLQR}

Replicate 1

$$
y=1.1 x+(18.7) \quad r . s q r=1
$$

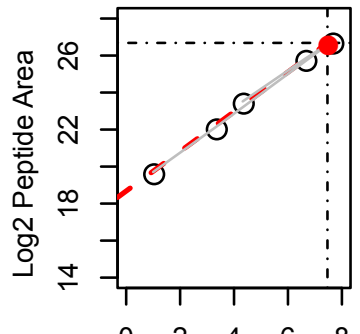

$\begin{array}{llll}0 & 2 & 4 & 6\end{array}$
Replicate 2

Replicate 3

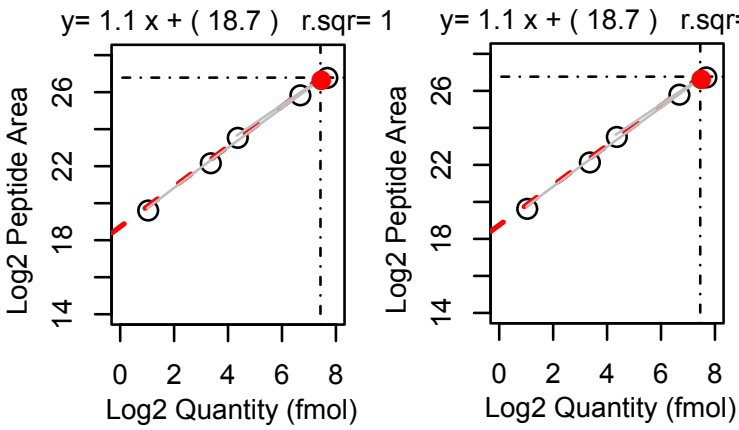


Replicate 1

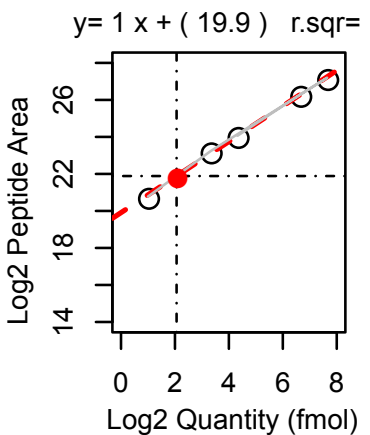

Replicate 2

Replicate 3

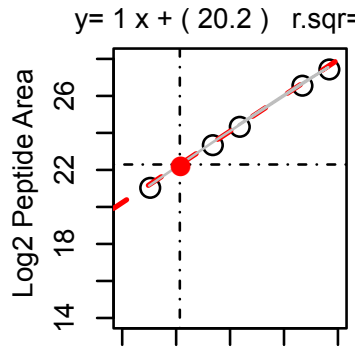

$\begin{array}{lllll}0 & 2 & 4 & 6 & 8\end{array}$ Log2 Quantity (fmol)

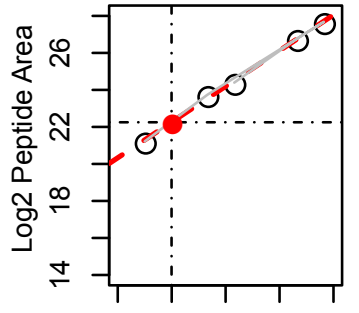

$\begin{array}{lllll}0 & 2 & 4 & 6 & 8\end{array}$ Log2 Quantity (fmol)

\section{0 fmol - ELVSEFSR}

Replicate 1

$y=1 x+(20.3) \quad r . s q r=1$

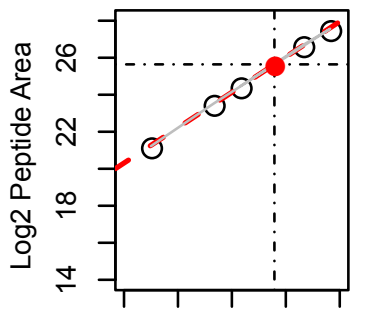

Log2 Quantity (fmol)
Replicate 2

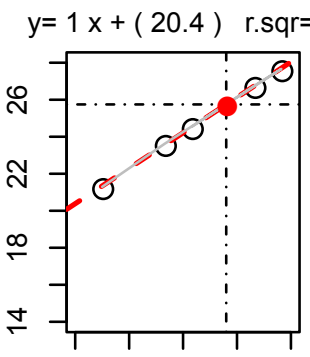

$\begin{array}{lllll}0 & 2 & 4 & 6 & 8\end{array}$ Log2 Quantity (fmol)
Replicate 3

$y=1 \mathrm{x}+(20.2) \quad$ r.sqr $=1$

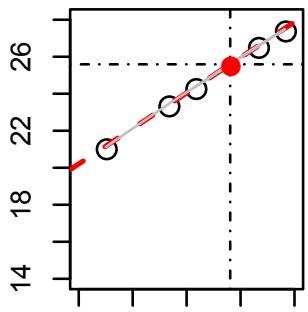

$\begin{array}{lllll}0 & 2 & 4 & 6 & 8\end{array}$ Log2 Quantity (fmol)

\section{0 fmol - ELVSEFSR}

Replicate 1

$$
y=1 x+(20.1) \quad r . s q r=1
$$

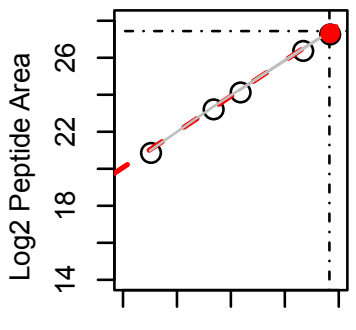

$\begin{array}{lllll}0 & 2 & 4 & 6 & 8\end{array}$ Log2 Quantity (fmol)
Replicate 2

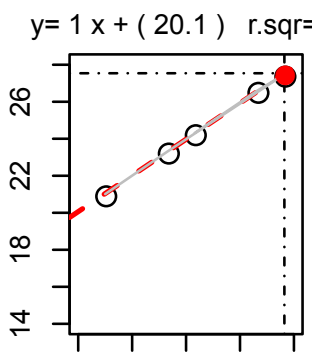

$\begin{array}{lllll}0 & 2 & 4 & 6 & 8\end{array}$ Log2 Quantity (fmol)
Replicate 3

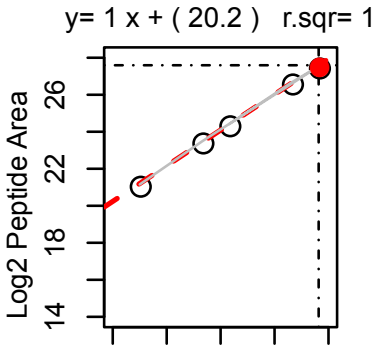

$\begin{array}{lllll}0 & 2 & 4 & 6 & 8\end{array}$ Log2 Quantity (fmol) 


\section{ELVSEFSR}

Patient S2

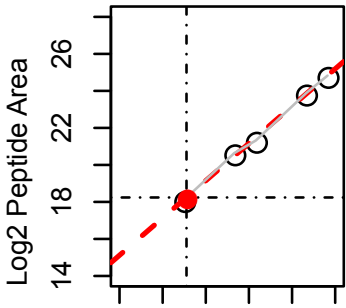

$\begin{array}{lllll}-2 & 2 & 4 & 6 & 8\end{array}$

Log2 Quantity (fmol)

Patient 58

$y=1 x+(16.7)$ r.sqr=

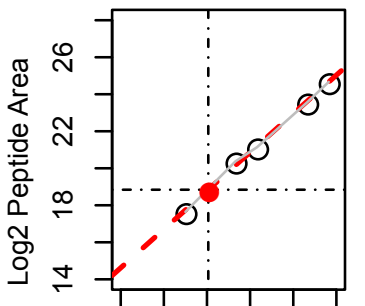

$\begin{array}{lllll}-2 & 2 & 4 & 6 & 8\end{array}$

Log2 Quantity (fmol)

Patient S14

$y=0.9 x+(16.6) \quad$ r.sqr

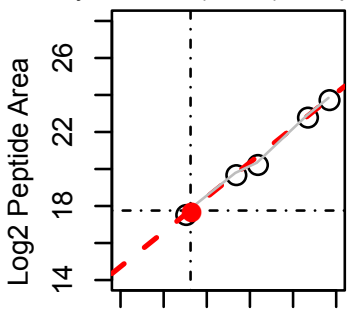

$\begin{array}{lllll}-2 & 2 & 4 & 6 & 8\end{array}$

Log2 Quantity (fmol)

Patient S20

$y=1 x+(17) \quad r . s q r=1$

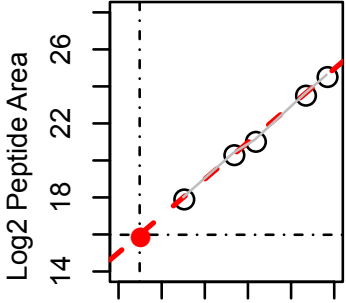

$\begin{array}{lllll}-2 & 2 & 4 & 6 & 8\end{array}$

Log2 Quantity (fmol)

Patient 526

$y=1 \mathrm{x}+(16.9) \quad$ r.sqr $=1$

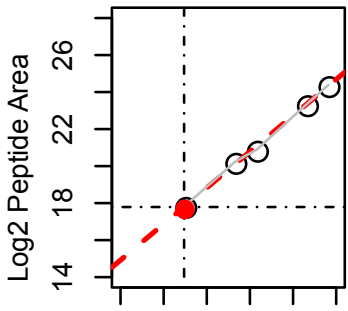

$\begin{array}{lllll}-2 & 2 & 4 & 6 & 8\end{array}$

Log2 Quantity (fmol)

Patient S32

$y=1 x+(18.4) \quad r . s q r=$

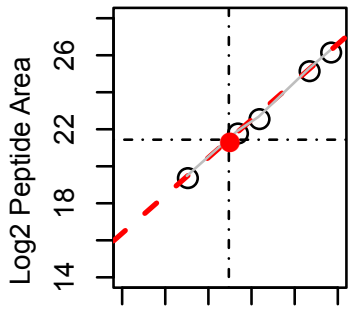

$\begin{array}{lllll}-2 & 2 & 4 & 6 & 8\end{array}$

Log2 Quantity (fmol)
Patient S4

Patient S5

Patient 56

Patient 57

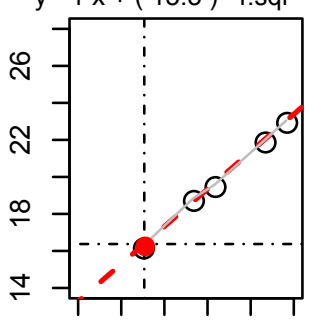

$\begin{array}{lllll}-2 & 2 & 4 & 6 & 8\end{array}$

Log2 Quantity (fmol)

Patient 59
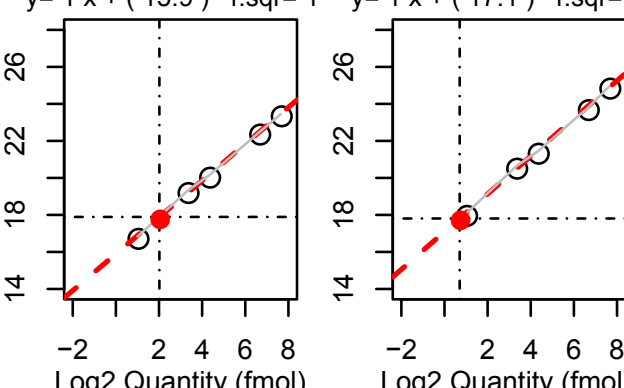

$\begin{array}{lllll}-2 & 2 & 4 & 6 & 8\end{array}$

Log2 Quantity (fmol)

Patient S10

Patient S11
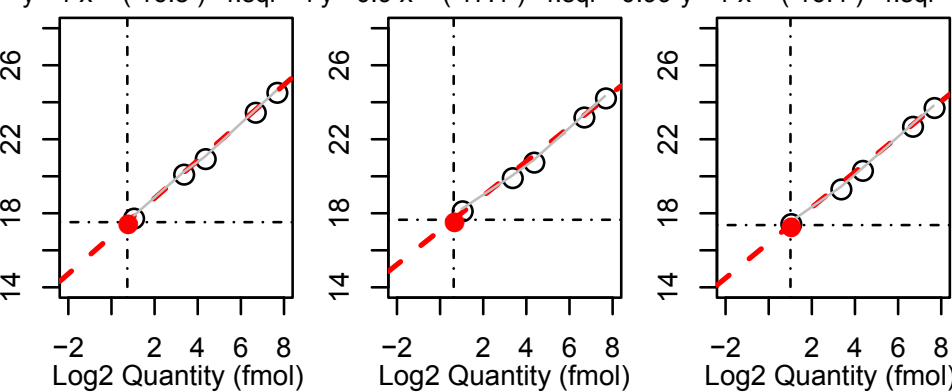

$\begin{array}{lllll}-2 & 2 & 4 & 6 & 8\end{array}$

Log2 Quantity (fmol)

Patient S15

Patient S16

Patient $\mathbf{S 1 7}$
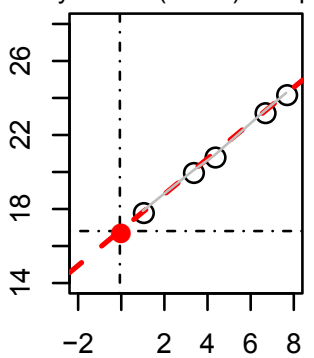

Log2 Quantity (fmol)

Patient S21

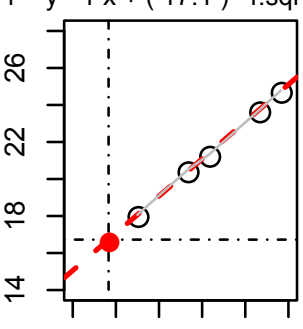

$\begin{array}{lllll}-2 & 2 & 4 & 6 & 8\end{array}$

Log2 Quantity (fmol)

Patient S22

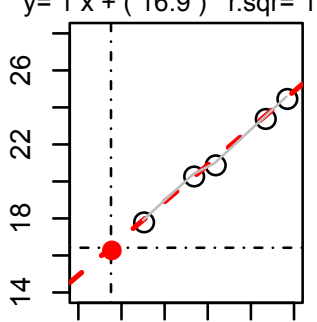

$\begin{array}{lllll}-2 & 2 & 4 & 6 & 8\end{array}$

Log2 Quantity (fmol)

Patient 527
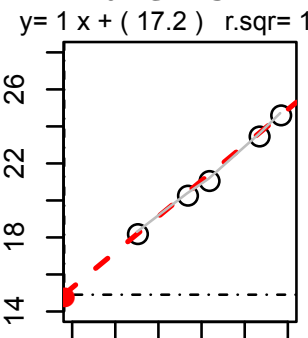

$\begin{array}{lllll}-2 & 2 & 4 & 6 & 8\end{array}$

Log2 Quantity (fmol)

Patient 528

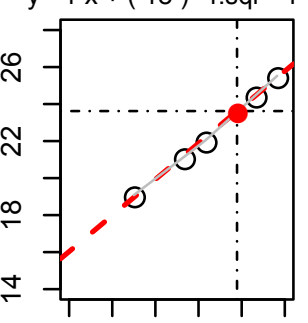

$\begin{array}{lllll}-2 & 2 & 4 & 6 & 8\end{array}$

Log2 Quantity (fmol)

Patient S33

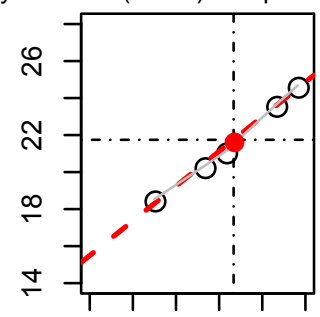

$\begin{array}{lllll}-2 & 2 & 4 & 6 & 8\end{array}$

Log2 Quantity (fmol)

Patient 534
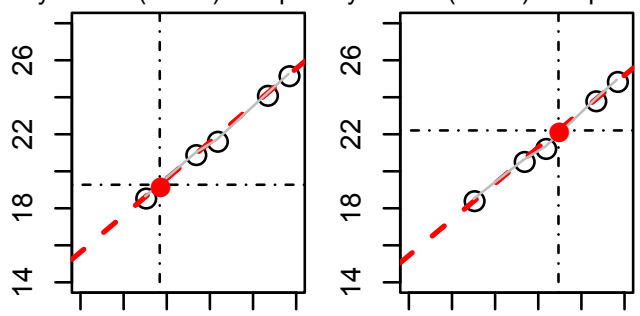

$\begin{array}{lllll}-2 & 2 & 4 & 6 & 8\end{array}$

$\begin{array}{lllll}-2 & 2 & 4 & 6 & 8\end{array}$

Log2 Quantity (fmol)

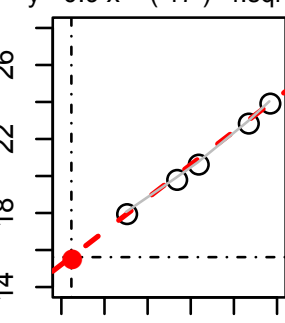

$\begin{array}{lllll}-2 & 2 & 4 & 6 & 8\end{array}$

Log2 Quantity (fmol)

Patient 523

$y=1 x+(16.8) \quad r . s q r=1$

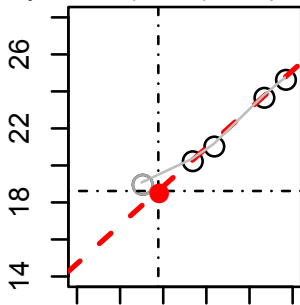

$\begin{array}{lllll}-2 & 2 & 4 & 6 & 8\end{array}$

Log2 Quantity (fmol)

Patient 529

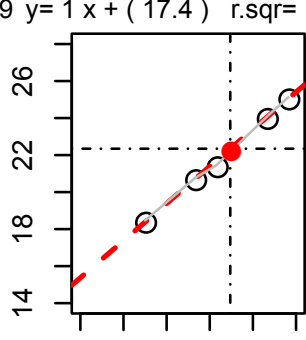

$\begin{array}{lllll}-2 & 2 & 4 & 6 & 8\end{array}$

Log2 Quantity (fmol)

Patient $\mathbf{5 3 5}$

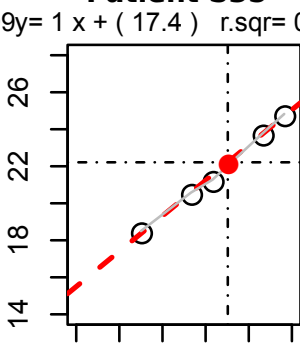

$\begin{array}{lllll}-2 & 2 & 4 & 6 & 8\end{array}$

Log2 Quantity (fmol)

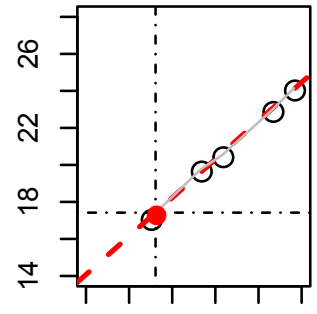

$\begin{array}{lllll}-2 & 2 & 4 & 6 & 8\end{array}$

Log2 Quantity (fmol)

Patient S12

$y=1 x+(17.2)$ r.sqr=1 $y=1.1 x+(16) \quad r . s q r=1$

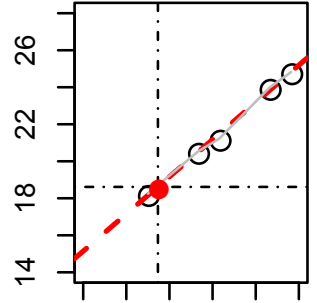

$\begin{array}{lllll}-2 & 2 & 4 & 6 & 8\end{array}$

Log2 Quantity (fmol)

Patient $\mathbf{S 1 8}$

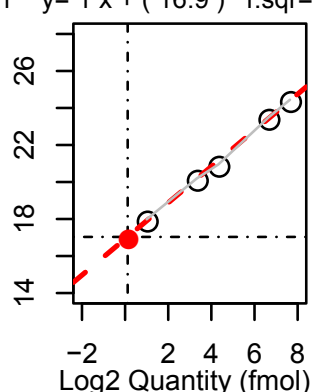

Patient 524
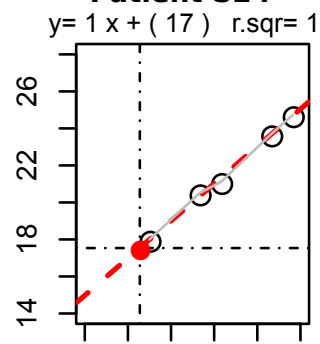

$\begin{array}{lllll}-2 & 2 & 4 & 6 & 8\end{array}$

Log2 Quantity (fmol)

Patient S30

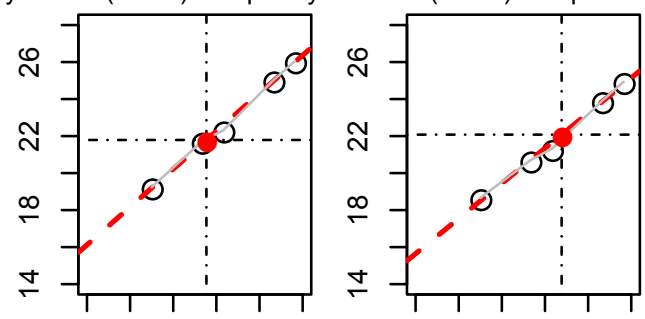

$\begin{array}{lllll}-2 & 2 & 4 & 6 & 8\end{array}$

Log2 Quantity (fmol)

Patient S36

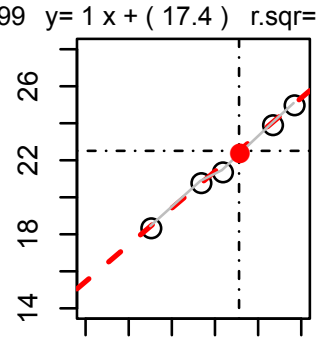

$\begin{array}{lllll}-2 & 2 & 4 & 6 & 8\end{array}$

Log2 Quantity (fmol)

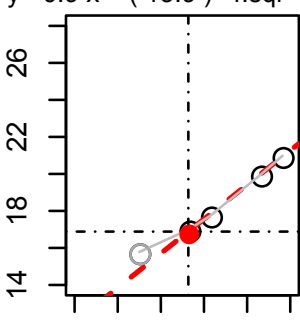

$\begin{array}{lllll}-2 & 2 & 4 & 6 & 8\end{array}$

Log2 Quantity (fmol)

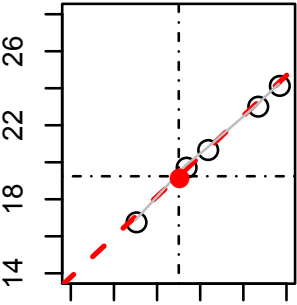

$\begin{array}{lllll}-2 & 2 & 4 & 6 & 8\end{array}$

Log2 Quantity (fmol)

Patient $\mathbf{S 1 9}$

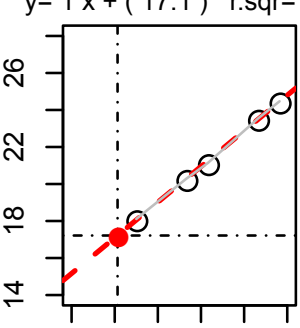

$\begin{array}{lllll}-2 & 2 & 4 & 6 & 8\end{array}$

Log2 Quantity (fmol)

Patient S25

$\mathrm{y}=1 \mathrm{x}+(16.7) \quad r . s q r=1$

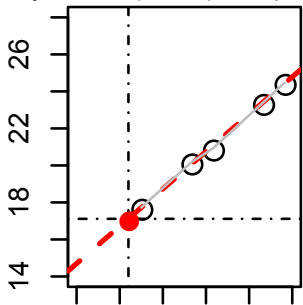

$\begin{array}{lllll}-2 & 2 & 4 & 6 & 8\end{array}$

Log2 Quantity (fmol)

Patient S31

$\begin{array}{lllll}-2 & 2 & 4 & 6 & 8\end{array}$ Log2 Quantity (fmol) 


\section{Supporting Information \\ Isotopologue multi-point calibration for proteomics biomarker quantification in clinical practice}

Cristina Chiva ${ }^{1,2}$, Olga Pastor ${ }^{1,2}$, Lucía Trilla-Fuertes ${ }^{3}$, Angelo Gámez-Pozo ${ }^{3,4}$, Juan Ángel Fresno Vara ${ }^{4}$, Eduard Sabidó ${ }^{1,2, *}$

${ }^{I}$ Proteomics Unit, Center for Genomics Regulation, Barcelona Institute of Science and Technology (BIST), 08003,

Barcelona, Spain

${ }^{2}$ Proteomics Unit, Universitat Pompeu Fabra, 08003, Barcelona, Spain

${ }^{3}$ Biomedica Molecular Medicine SL, C/ Faraday 7, 28049, Madrid, Spain

${ }^{4}$ Molecular Oncology \& Pathology Lab, Institute of Medical and Molecular Genetics-INGEMM, La Paz

University Hospital-IdiPAZ, Paseo de la Castellana 261, 28046, Madrid, Spain

* Corresponding author

Eduard Sabidó

Proteomics Unit, Center for Genomics Regulation, Barcelona Institute of Science and Technology (BIST),

Universitat Pompeu Fabra, Barcelona, Spain

Dr. Aiguader 88

08003 Barcelona

eduard.sabido@crg.cat

\section{Table of Contents}

Supplementary Figures S1-S4

Supplementary Tables S1-S6

Supplementary Methods

- Isotopologue peptides

- Background matrix preparation

- Calibration curves preparation

- Control sample preparation

- Formalin-fixed paraffin-embedded sample preparation

- Data acquisition

- Bibliography 


\section{Supplementary Figures}
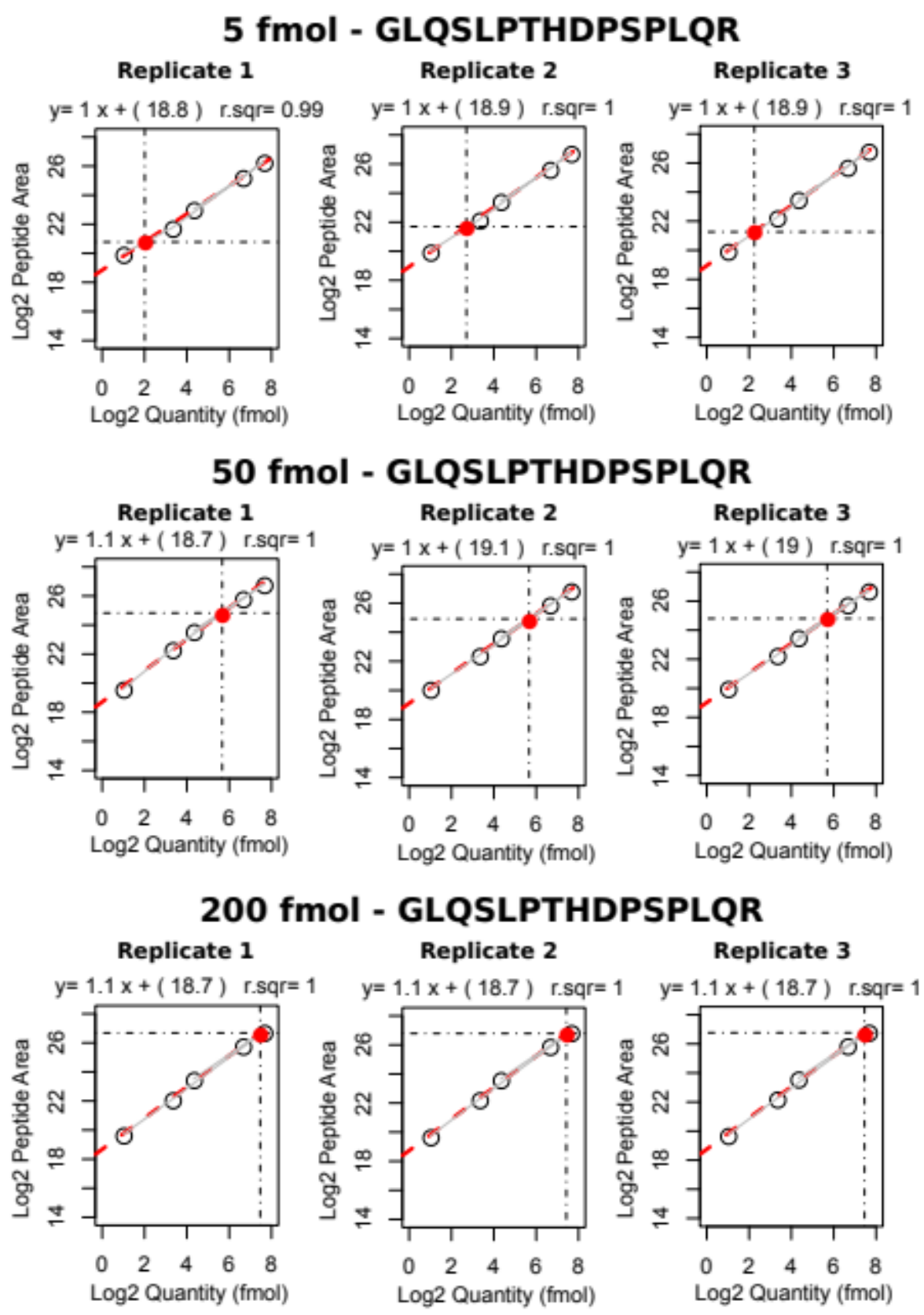

Figure S1: Calculated abundance of peptide ELVSEFSR in controlled samples (5, 50, 200 fmol) using the Isotopologue Multiple-point Calibration ( $\mathrm{ImCal}$ ) quantification strategy in three independent replicates. The logarithmic area for the different quantities of all the isotopologue peptides are indicated with empty circles. The dashed red line is the result of the linear regression model (see equation in each plot), whereas the light gray is a polynomial fit to identify devitations from linearity. The red dot indicates the area and quantity of the endogenous peptide estimated using the linear regression model. 


\section{5 fmol - ELVSEFSR}
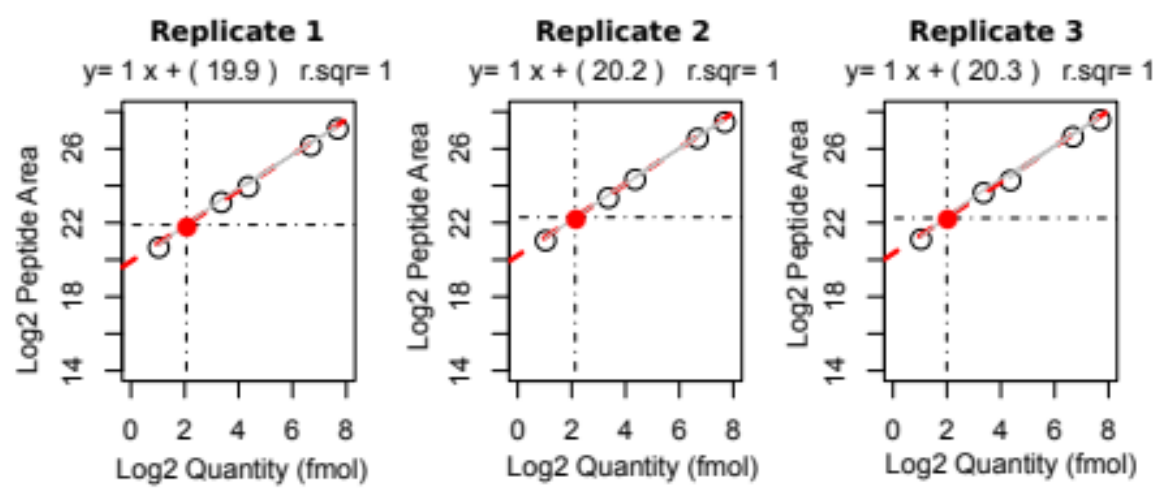

\section{0 fmol - ELVSEFSR}

Replicate 1 $y=1 x+(20.3) \quad r . s q r=1$

Replicate 2

$y=1 x+(20.4) \quad r . s q r=1$
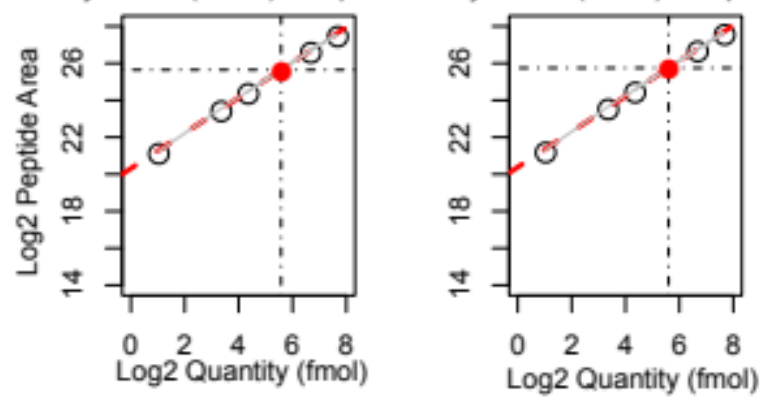

Replicate 3

$y=1 \mathrm{x}+(20.2) \quad r . s q r=1$

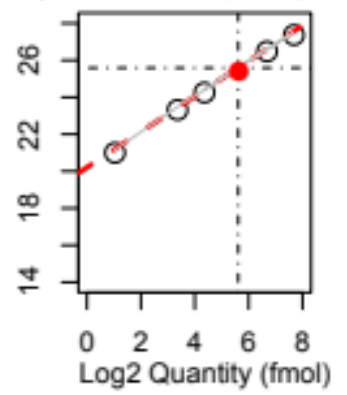

Replicate 3

Replicate 1

$200 \mathrm{fmol}$ - ELVSEFSR

Replicate 2

$y=1 x+(20.1) \quad r . s q r=1$

$y=1 x+(20.2) \quad r . s q r=1$
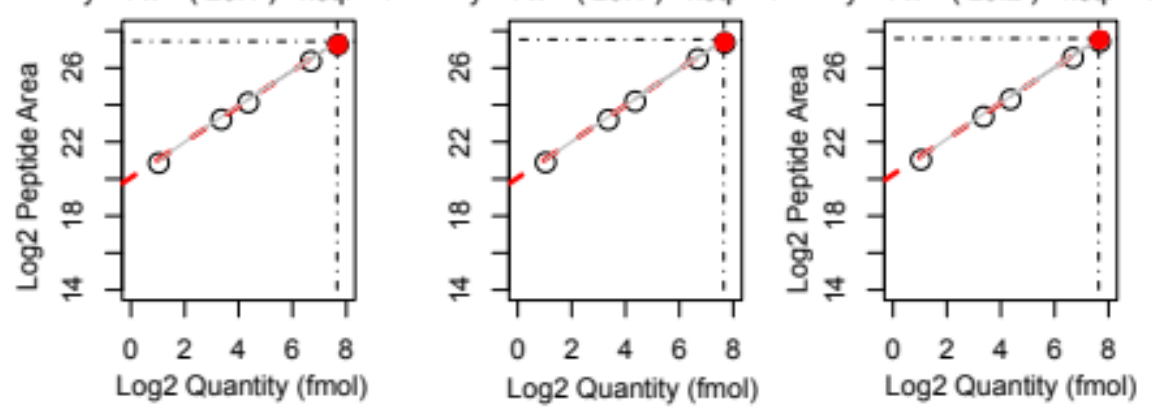

Figure S2 Calculated abundance of peptide GLQSLPTHDPSPLQR in controlled samples (5, 50, 200 fmol) using the Isotopologue Multiple-point Calibration (ImCal) quantification strategy in three independent replicates. The logarithmic area for the different quantities of all the isotopologue peptides are indicated with empty circles. The dashed red line is the result of the linear regression model (see equation in each plot), whereas the light gray is a polynomial fit to identify devitations from linearity. The red dot indicates the area and quantity of the endogenous peptide estimated using the linear regression model. 


\section{ELVSEFSR}
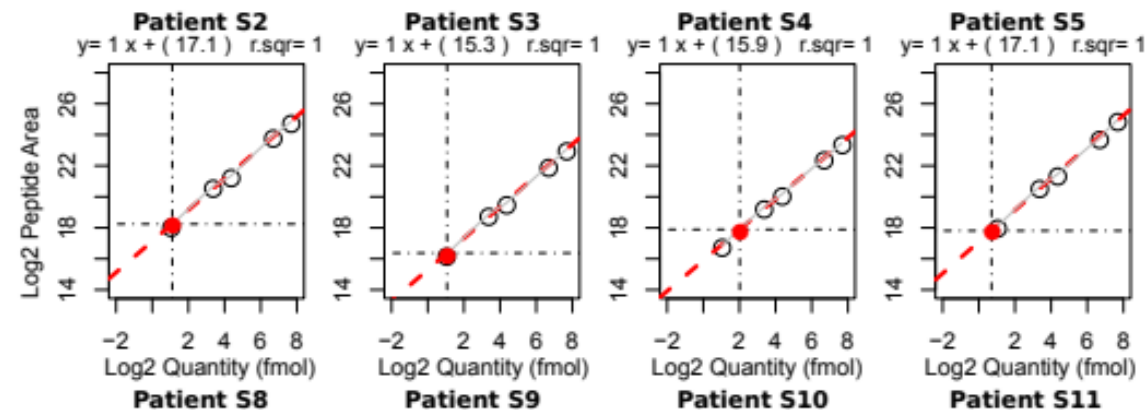

Patient S6

Patient 57
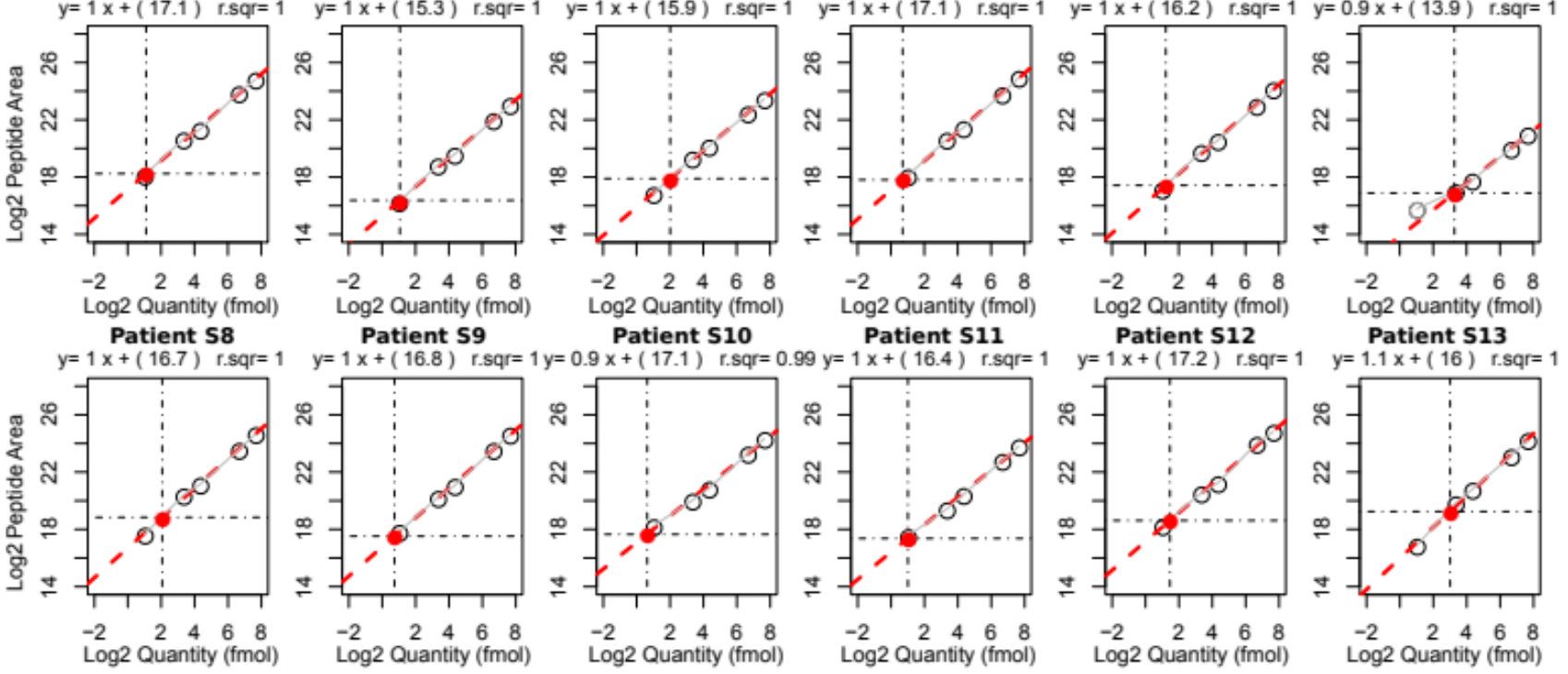

Patient 511
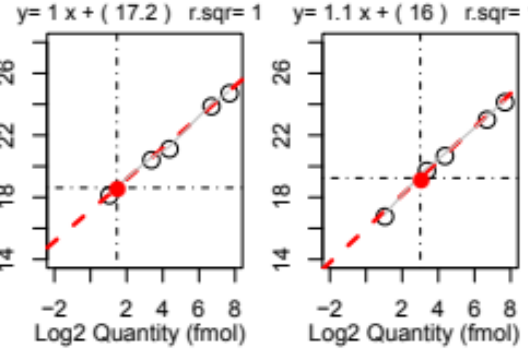

Patient 514

Patient S15

Patient $\mathbf{S 1 6}$

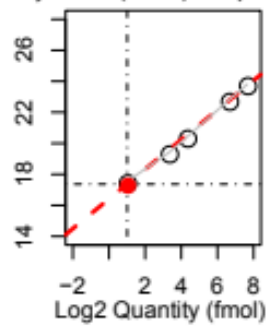

Patient $\mathbf{5 1 8}$

Patient 519
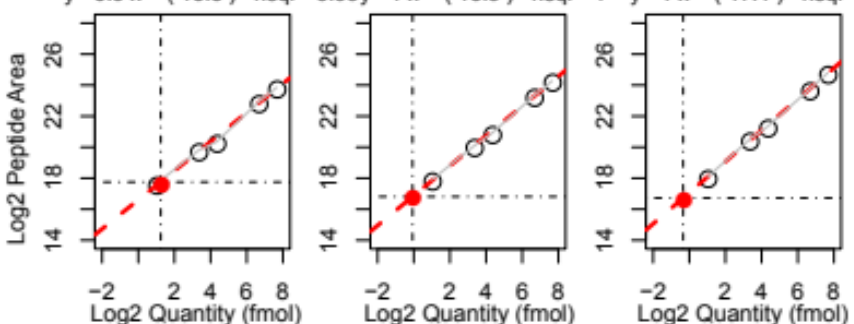

Patient S17
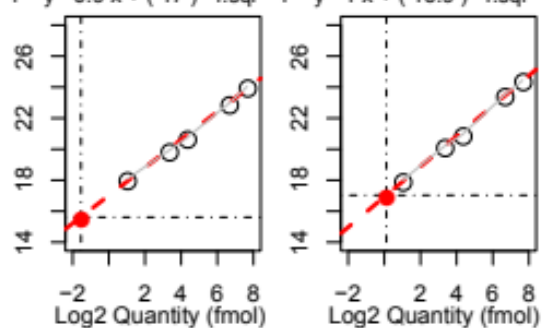

$\begin{array}{lllll}-2 & 2 & 4 & 6 & 8\end{array}$

$\begin{array}{lllll}-2 & 2 & 4 & 6 & 8\end{array}$

Patient S22

Patient $\mathbf{5 2 3}$

$\begin{array}{lllll}-2 & 2 & 4 & 6 & 8\end{array}$

Log2 Quantity (fmol)

Patient 524

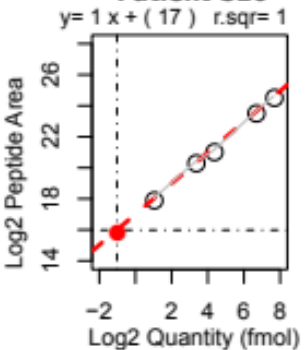

Patient S21

$y=1 x+(17.2) \quad r . s q r=1$
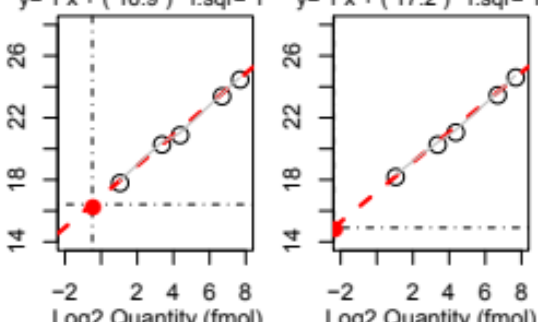

$y=1 x+(16.8) \quad r . s q r=1$

$y=1 x+(17) \quad r . s q r=1$

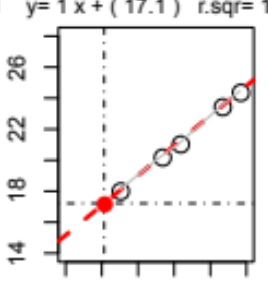

$\begin{array}{lllll}-2 & 2 & 4 & 6 & 8\end{array}$

og2 Quantity (fmol)

Patient S25
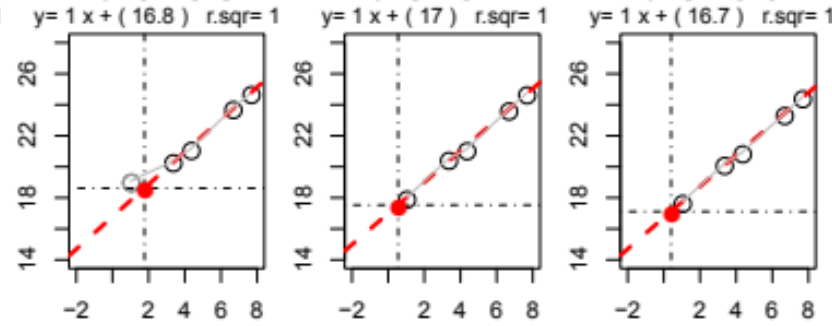

Patient S26

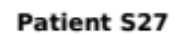

Patient 528

Patient 529

$\begin{array}{lllll}-2 & 2 & 4 & 6 & 8 \\ \text { Log2 } & \text { Quantity (fmol) }\end{array}$

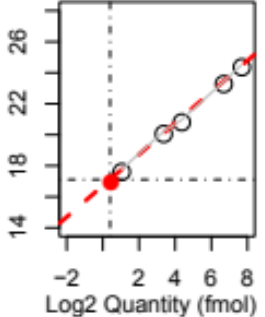

Patient S30

Patient $\mathbf{5 3 1}$
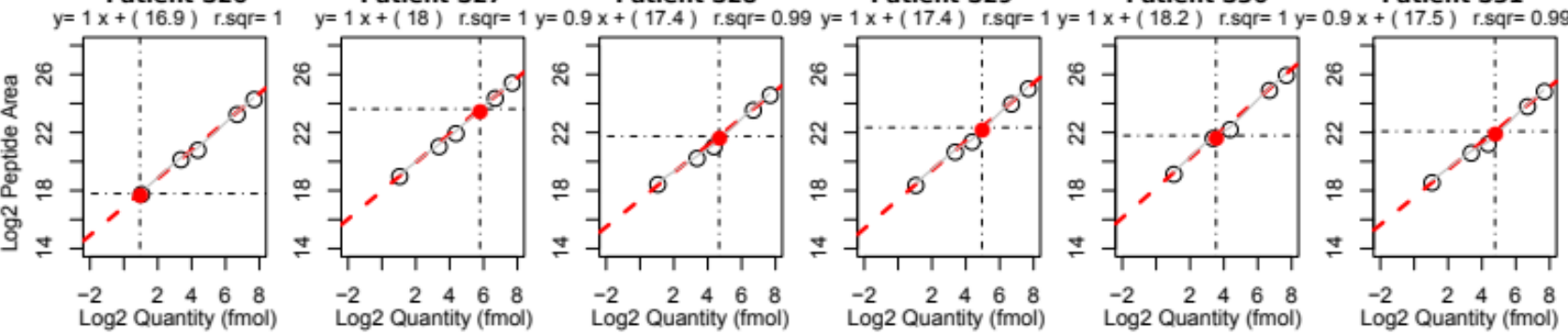

Patient S32

Patient S33

Patient S34

Patient $\mathbf{5 3 5}$

Log2 Quantity (fmol)

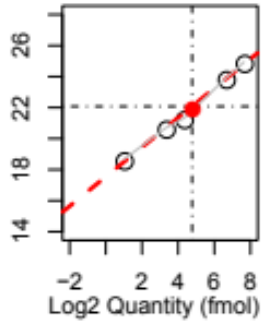

$y=1 x+(18.4) \quad r . s q r=$
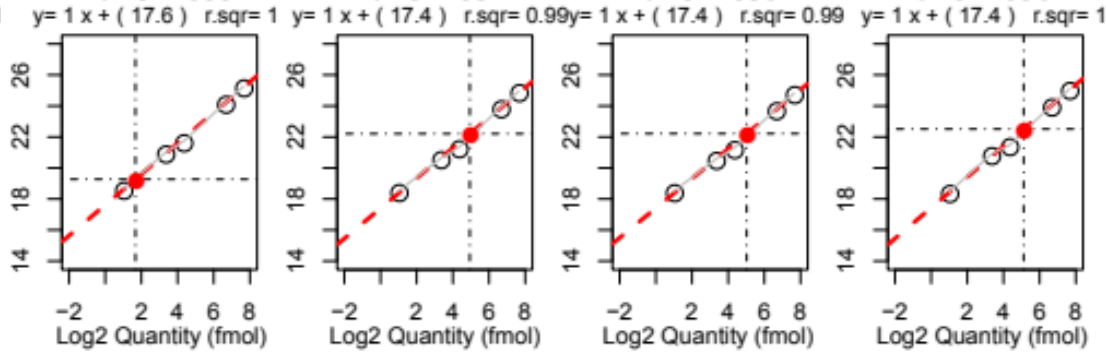
Figure S3 Calculated abundance of peptide ELVSEFSR in FFPE samples from Her2+ and Her2- patients using the Isotopologue Multiple-point Calibration ( $\mathrm{ImCal}$ ) quantification strategy. The logarithmic area for the different quantities of all the isotopologue peptides are indicated with empty circles. The dashed red line is the result of the linear regression model (see equation in each plot), whereas the light gray is a polynomial fit to identify devitations from linearity. The red dot indicates the area and quantity of the endogenous peptide estimated using the linear regression model. 


\section{GLQSLPTHDPSPLQR}

Patient S2 $\begin{array}{lllll}-2 & 2 & 4 & 6 & 8 \\ \log 2 & \text { Quantity (fmol) }\end{array}$

Patient $\mathbf{5 8}$

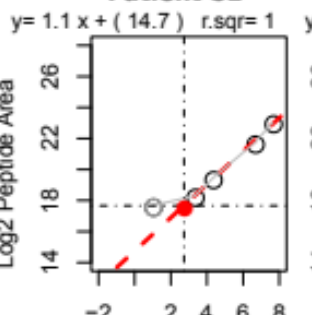

Patient S3

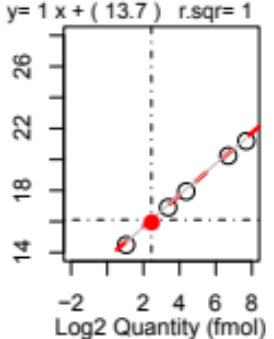

Patient S9
Patient S4

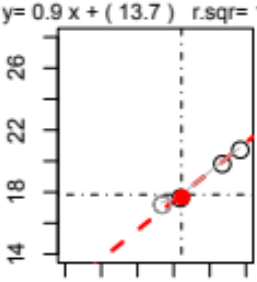

$\begin{array}{lllll}-2 & 2 & 4 & 6 & 8\end{array}$ Log2 Quantity (fmol) Patient S10
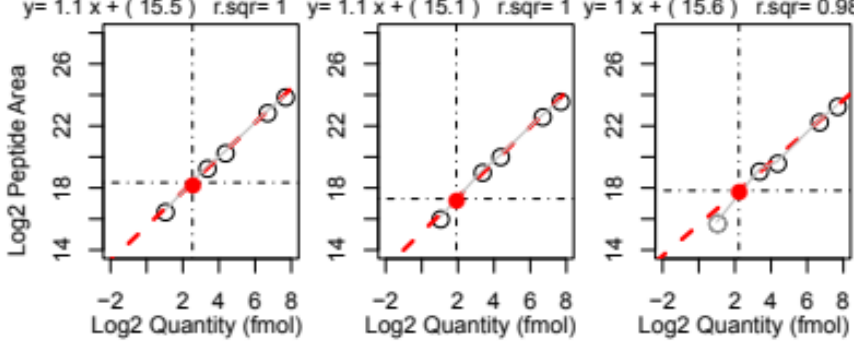

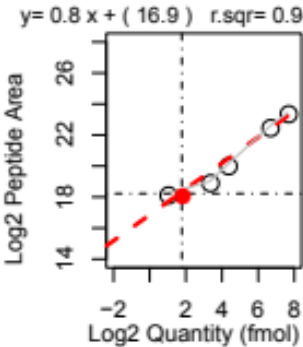

Log2 Quantity (fmol)

Patient S20

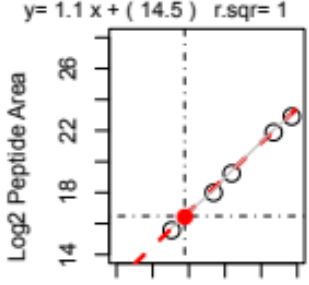

$\begin{array}{llllll}-2 & 2 & 4 & 6 & 8\end{array}$ Log2 Quantity (fmol)

Patient S26

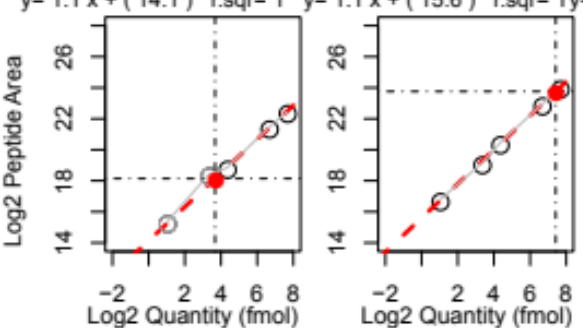

Patient S32

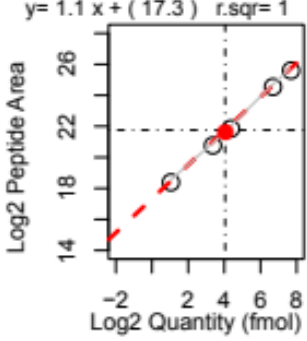

Patient S15

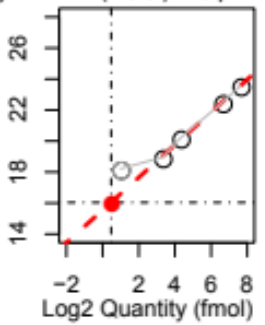

Patient S21

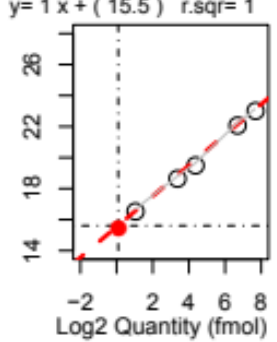

Patient S27

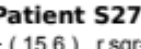

Patient S16

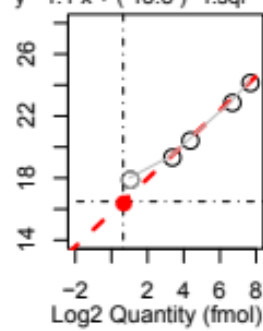

Patient $\mathbf{5 2 2}$

$y=1 x+(15.3) \quad$ r.sqr $=1$

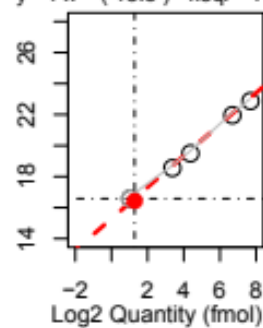

Patient $\mathbf{5 2 8}$

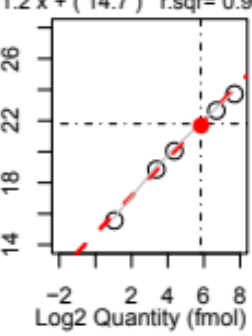

Patient S34
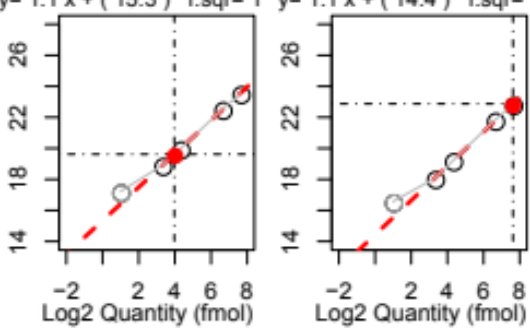

Patient 55

Patient S6

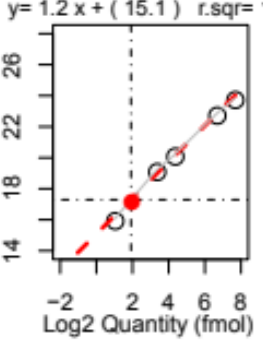

Patient 511

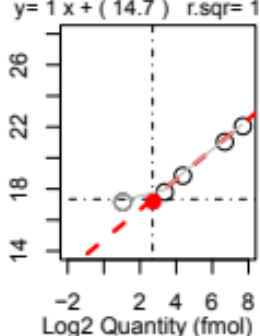

Patient

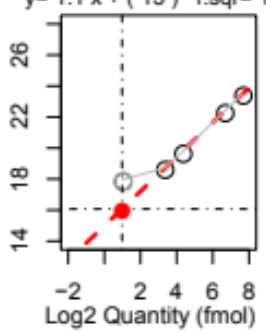

Patient S23

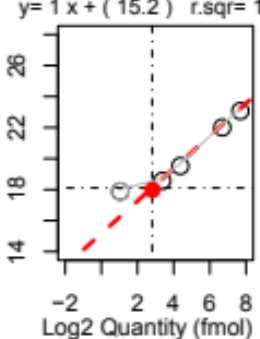

Patient 529

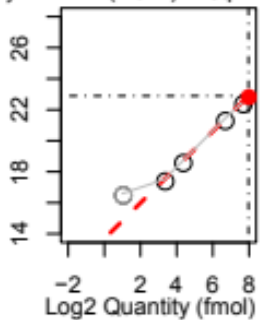

Patient S35

Patient 57

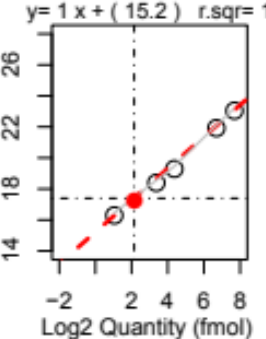

og2 Quantity (fmol)

Patient $\mathbf{S 1 2}$

(x) $x+(16.3) \quad r . s q r=0.99 y=1.1 x$

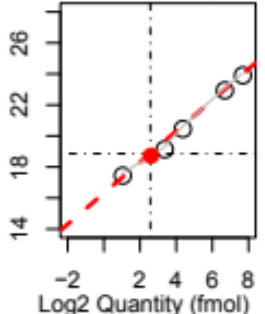

Patient $\mathbf{S 1 8}$

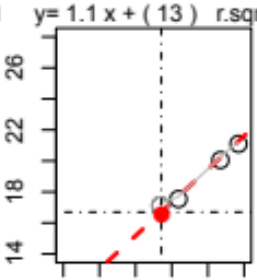

$\begin{array}{lllll}-2 & 2 & 4 & 6 & 8\end{array}$

Log2 Quantity (fmol)

Patient S13

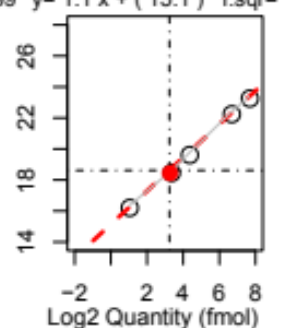

Patient S19
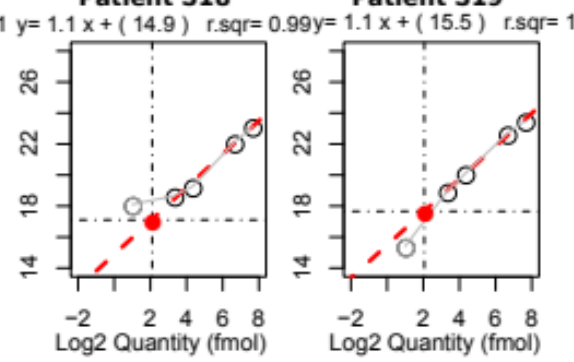

Patient S24

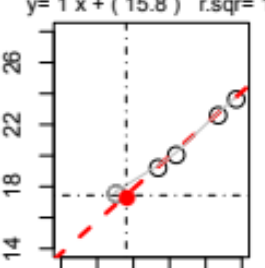

Patient $\mathbf{5 2 5}$

$y=1 x+(14.8) \quad r . s q r=1$

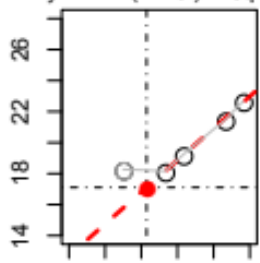

$\begin{array}{lllll}-2 & 2 & 4 & 6 & 8\end{array}$

Patient S30

$\begin{array}{lllll}-2 & 2 & 4 & 6 & 8\end{array}$

Patient S31

$=1.1 \mathrm{x}+(16.3)$ r.sqr $=$
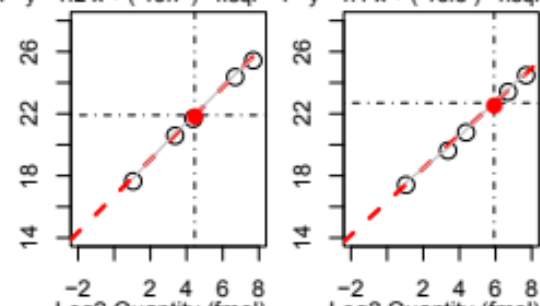

Patient $\mathbf{5 3 6}$

$1.2 x+(14.9) \quad r . s q r=$

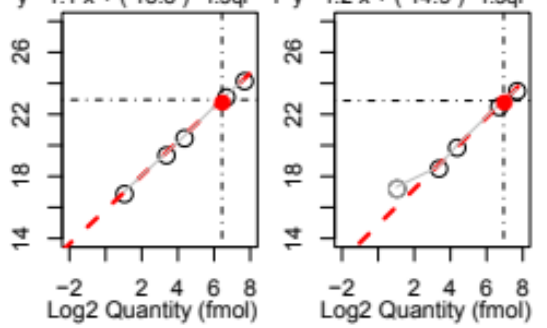


Figure S4 Calculated abundance of peptide GLQSLPTHDPSPLQR in FFPE samples from Her2+ and Her2patients using the Isotopologue Multiple-point Calibration ( $\mathrm{ImCal}$ ) quantification strategy. The logarithmic area for the different quantities of all the isotopologue peptides are indicated with empty circles. The dashed red line is the result of the linear regression model (see equation in each plot), whereas the light gray is a polynomial fit to identify devitations from linearity. The red dot indicates the area and quantity of the endogenous peptide estimated using the linear regression model. 


\section{Supplementary Tables}

Table S1: Targeted proteomics results for the generation of the reference external calibration curve using selected reaction monitoring.

Table S2: Targeted proteomics results for the generation of the reference external reverse calibration curve using selected reaction monitoring.

Table S3: Targeted proteomics results for the quantification of 5, 50, 200 fmol of peptides ELVSEFSR and GLQSLPTHDPSPLQR in the controlled samples using selected reaction monitoring.

Table S4: Calculated abundances of peptides ELVSEFSR and GLQSLPTHDPSPLQR in controlled samples using different calibration strategies.

Table S5: Targeted proteomics results for the quantification of endogenous peptides ELVSEFSR and GLQSLPTHDPSPLQR in FFPE samples from Her2+ and Her2- patients using the Isotopologue Multiple-point Calibration ( $\mathrm{ImCal}$ ) quantification strategy and selected reaction monitoring.

Table S6: Calculated abundances of endogenous peptides ELVSEFSR and GLQSLPTHDPSPLQR in FFPE samples from Her2+ and Her2- patients using the Isotopologue Multiple-point Calibration (ImCal) quantification. 


\section{Supplementary Methods}

\section{Isotopologue peptides}

Five HVYPEP AQUA Ultimate isotopologues peptides (Thermo Fisher Scientific, Waltham, MA, USA) were ordered for each of the two selected peptides from the human Receptor tyrosine-protein kinase erbB-2 (ELVSEFSR and GLQSLPTHDPSPLQR; HER2, P04626) using different combinations of the following isotopically-labelled amino acids 13C615N4-Arg (+10), 13C515N1-Val (+6), 13C615N1-Leu (+7), 13C915N1-Phe (+10), 13C515N1Pro $(+6)$ and 13C515N1-Glu (+6). The isotopologue peptides synthesized were ELVSEFSR $(+0)$, ELVSEFSR* $(+10), \mathrm{ELV}^{*} \mathrm{SEFSR}^{*}(+16), \mathrm{EL} * \mathrm{~V}^{*} \mathrm{SEFSR}^{*}(+23), \mathrm{EL}^{*} \mathrm{~V} * \mathrm{SEF} * \mathrm{SR} *(+33), \mathrm{E}^{*} \mathrm{~L}^{*} \mathrm{~V} * \mathrm{SEF} * \mathrm{SR} *(+39)$; and GLQSLPTHDPSPLQR (+0), GLQSLPTHDPSPLQR* (+10), GLQSLPTHDPSPL*QR* (+17), GLQSLPTHDP*SPL*QR* (+23), GLQSL*PTHDP*SPL*QR* (+30), GL*QSL*PTHDP*SPL*QR* (+37).

\section{Background matrix preparation}

A protein extract from E. coli was used as background matrix for the preparation of calibration curves and control samples. Briefly, $2.7 \mathrm{mg}$ of a commercial E. coli protein extract (P/N 1632110, Bio-Rad, Hercules, CA, USA) were reduced with $3 \mathrm{mM}$ dithiothreitol, alkylated with $6 \mathrm{mM}$ iodoacetamide, and sequentially digested with Lysyl endopeptidase LysC (Wako, Japan) at 1:10 ratio (w:w; enzyme:substrate) at $37^{\circ} \mathrm{C}$ overnight, and trypsin (Promega, Madison, WI, USA) 1:10 ratio (w:w; enzyme:substrate) at $37{ }^{\circ} \mathrm{C}$ for 8 hours. Digested peptide mixtures were desalted using a C18 Hypersep column (Thermo Fisher Scientific).

\section{Calibration curves preparation}

Internal calibration curves for the controlled experiments were prepared with different amounts of isotopologue peptides ranging from 2 to $200 \mathrm{fmol} / \mu \mathrm{L}$. $1 \mathrm{X}$ internal calibration curve consisted on $2 \mathrm{fmol} / \mu \mathrm{L}$ of $\mathrm{E}^{*} \mathrm{~L} * \mathrm{~V} * \mathrm{SEF} * \mathrm{SR}^{*}$ and $\mathrm{GL}^{*} \mathrm{QSL} * \mathrm{PTHDP} * \mathrm{SPL} * \mathrm{QR} *, 10 \mathrm{fmol} / \mu \mathrm{L}$ of EL*V*SEF*SR* and GLQSL*PTHDP*SPL*QR*, $20 \mathrm{fmol} / \mu \mathrm{L}$ of ELVSEFSR* and GLQSLPTHDPSPLQR*, $100 \mathrm{fmol} / \mu \mathrm{L}$ of ELV*SEFSR* and GLQSLPTHDPSPL*QR*, and $200 \mathrm{fmol} / \mu \mathrm{L}$ of EL*V*SEFSR* and GLQSLPTHDP*SPL*QR*,

External calibration curves were build injecting $1 \mu \mathrm{L}$ of a mix containing the non-labeled peptides (ELVSEFSR and GLQSLPTHDPSPLQR) in a given amount ( $0 \mathrm{fmol}, 2 \mathrm{fmol}, 10 \mathrm{fmol}, 20 \mathrm{fmol}, 100 \mathrm{fmol}$ and $200 \mathrm{fmol}$ ), together with a fixed amount of isotope-labeled peptides $\left(20 \mathrm{fmol}\right.$ of ELVSEFSR ${ }^{*}$ and 20 fmol of GLQSLPTHDPSPLQR ${ }^{*}$ ), and $1 \mu \mathrm{g}$ of digested E. coli protein extract (see above). Each point of the calibration curve was done in triplicate.

Finally, reverse external calibration curves were build injecting $1 \mu \mathrm{L}$ of a mix containing the isotopically-labelled peptides ELVSEFSR* and GLQSLPTHDPSPLQR* in a given amount ( $0 \mathrm{fmol}, 2 \mathrm{fmol}, 10 \mathrm{fmol}, 20 \mathrm{fmol}, 100 \mathrm{fmol}$ and $200 \mathrm{fmol}$ ) together with a fixed amount of non-labeled peptides (20 fmol of ELVSEFSR and $20 \mathrm{fmol}$ of GLQSLPTHDPSPLQR) and $1 \mu \mathrm{g}$ of digested E. coli protein extract (see above). Each point of the calibration curve was done in triplicate.

\section{Control sample preparation}

Four control samples were prepared with $1 \mathrm{X}$ internal calibration curve, $1 \mu \mathrm{g} / \mu \mathrm{L}$ of $E$. coli digested protein extract (see above), and either 5, 50 or $200 \mathrm{fmol} / \mu \mathrm{L}$ of the commercial non-labeled peptides (ELVSEFSR and GLQSLPTHDPSPLQR). Control samples were prepared in triplicate.

\section{Formalin-fixed paraffin-embedded sample preparation}

Formalin-fixed paraffin-embedded (FFPE) samples from patients diagnosed of triple negative and HER2 positive breast cancer were retrieved from IdiPAZ Biobank (RD09/0076/00073), which is integrated in the Spanish 
Hospital Biobank Network (RetBioH; www.redbiobancos.es). The histopathological features of each sample were reviewed by an experienced pathologist to confirm diagnosis and tumor content. Eligible samples had to include at least $50 \%$ of tumor cells. Written consent was provided by all patients participating in this study, and approval from the Ethical Committees of Hospital La Paz was obtained for the conduct of the study.

Proteins were extracted from FFPE samples as previously described. ${ }^{1}$ Briefly, FFPE sections were deparaffinized in xylene and washed twice with ethanol. Protein extracts from FFPE samples were prepared in 2\% SDS buffer using a protocol based on heat-induced antigen retrieval. ${ }^{2}$ Protein concentration was determined using the MicroBCA Protein Assay Kit (ThermoFisher Scientific). Protein extracts $(\sim 10 \mu \mathrm{g})$ were digested with trypsin (1:50) and SDS was removed from digested lysates using Detergent Removal Spin Columns (ThermoFisher Scientific). Peptide samples were further desalted, dried, and solubilized in $0.1 \%$ formic acid and $3 \%$ acetonitrile solution before MS analysis, and isotopically-labeled peptides were spiked in the peptide mixtures and used as multi-point internal standard for quantification with selected reaction monitoring (SRM). The mixture of the isotopically-labelled peptides consisted in $2 \mathrm{fmol} / \mu \mathrm{L}$ of E* $\mathrm{L}^{*} \mathrm{~V}^{*} \mathrm{SEF} * \mathrm{SR}^{*}$ and GL*QSL*PTHDP*SPL*QR ${ }^{*}, 10$ $\mathrm{fmol} / \mu \mathrm{L}$ of $\mathrm{EL} * \mathrm{~V} * \mathrm{SEF}^{*} \mathrm{SR} *$ and GLQSL*PTHDP*SPL*QR*, $20 \mathrm{fmol} / \mu \mathrm{L}$ of EL*V*SEFSR and GLQSLPTHDP*SPL*QR*, $100 \mathrm{fmol} / \mu \mathrm{L}$ of ELV*SEFSR* and GLQSLPTHDPSPL*QR*, and $200 \mathrm{fmol} / \mu \mathrm{L}$ of ELVSEFSR* and GLQSLPTHDPSPLQR*.

\section{Data acquisition}

SRM measurements were performed on a 5500 Q-Trap mass spectrometer (Sciex, Framingham, MA, USA) coupled to a nanoLC Ultra-1DPlus (Sciex). Briefly, peptide mixtures were loaded onto a C18 Acclaim PepMap precolumn (cat \# 164564, Thermo Fisher Scientific) and were separated by reversed-phase chromatography using a $25-\mathrm{cm}$ column with an inner diameter of $75 \mu \mathrm{m}$, and packed with $1.9 \mu \mathrm{m}$ C18 particles (Nikkyo Technos Co, Japan). The chromatographic gradient started at $98 \%$ buffer A $(0.1 \%$ formic acid in water $)$ and $2 \%$ buffer B $(0.1 \%$ formic acid in acetonitrile) with a flow rate of $250 \mathrm{nl} / \mathrm{min}$ for $5 \mathrm{~min}$ and gradually increased to $60 \%$ buffer A and $40 \%$ buffer B in $35 \mathrm{~min}$. After each analysis, both the pre-column and the column were washed for 10 min with $2 \%$ buffer A and $98 \%$ buffer B. Digested bovine serum albumin was analyzed between the SRM measurements of biological samples to avoid sample carryover and to assure stability of the instrument. Measurements were done in non-scheduled SRM mode with dwell times of $10 \mathrm{~ms}$ per transition and a total cycle time of $\sim 2$ seconds. QCloud has been used to control instrument longitudinal performance during the project. ${ }^{3}$ The targeted proteomics data was analyzed with Skyline v4.1 and data have been deposited to the Panorama Public ${ }^{4,5}$ with the dataset identifier PXD012035. 


\section{Bibliography}

(1) Gámez-Pozo, A.; Ferrer, N. I.; Ciruelos, E.; López-Vacas, R.; Martínez, F. G.; Espinosa, E.; Vara, J. Á. F. Shotgun Proteomics of Archival Triple-Negative Breast Cancer Samples. PROTEOMICS-Clinical Appl. 2013, 7 (3-4), 283-291.

(2) Gámez-Pozo, A.; Sánchez-Navarro, I.; Calvo, E.; Díaz, E.; Miguel-Martín, M.; López, R.; Agulló, T.; Camafeita, E.; Espinosa, E.; López, J. A. Protein Phosphorylation Analysis in Archival Clinical Cancer Samples by Shotgun and Targeted Proteomics Approaches. Mol. Biosyst. 2011, 7 (8), 2368-2374.

(3) Chiva, C.; Olivella, R.; Borràs, E.; Espadas, G.; Pastor, O.; Solé, A.; Sabidó, E. QCloud: A Cloud-Based Quality Control System for Mass Spectrometry-Based Proteomics Laboratories. PLoS ONE 2018, 13 (1). https://doi.org/10.1371/journal.pone.0189209.

(4) Sharma, V.; Eckels, J.; Schilling, B.; Ludwig, C.; Jaffe, J. D.; MacCoss, M. J.; MacLean, B. Panorama Public: A Public Repository for Quantitative Data Sets Processed in Skyline. Mol. Cell. Proteomics 2018, 17 (6), $1239-1244$.

(5) Vizcaíno, J. A.; Deutsch, E. W.; Wang, R.; Csordas, A.; Reisinger, F.; Ríos, D.; Dianes, J. A.; Sun, Z.; Farrah, T.; Bandeira, N.; et al. ProteomeXchange Provides Globally Coordinated Proteomics Data Submission and Dissemination. Nat. Biotechnol. 2014, 32 (3), 223-226. https://doi.org/10.1038/nbt.2839. 